\title{
Mining of candidate genes involved in the biosynthesis of dextrorotatory borneol in Cinnamomum burmannii by transcriptomic analysis on three chemotypes
}

\author{
Zerui Yang ${ }^{1}$, Wenli An ${ }^{1}$, Shanshan Liu ${ }^{1}$, Yuying Huang ${ }^{1}$, Chunzhu Xie ${ }^{1}$, Song Huang ${ }^{\text {Corresp., }}{ }^{1}$, Xiasheng Zheng ${ }^{\text {Corresp. } 1}$ \\ ${ }^{1}$ National Engineering Research Center for Modernization of Traditional Chinese Medicine, Mathematical Engineering Academy of Chinese Medicine, \\ Guangzhou University of Chinese Medicine, Guangzhou, Guangdong, China \\ Corresponding Authors: Song Huang, Xiasheng Zheng \\ Email address: huangnn421@163.com, xszheng@gzucm.edu.cn
}

Background: Dextrorotatory borneol (D-borneol), a cyclic monoterpene, is widely used in traditional Chinese medicine as an efficient topical analgesic drug. Fresh leaves of Cinnamomum trees, e.g. C. burmannii and $C$. camphor, are the main sources from which D-borneol is extracted by steam distillation, yet with low yields. Insufficient supply of D-borneol has hampered its clinical use and production of patent remedies for a long time. Biological synthesis of D-borneol offers an additional approach; however, mechanisms of $\mathrm{D}$-borneol biosynthesis remain mostly unresolved. Hence, it is important and necessary to elucidate the biosynthetic pathway of D-borneol.

Results: Comparative analysis on the gene expression patterns of different D-borneol production $C$. burmannii samples facilitates elucidation on the underlying biosynthetic pathway of D-borneol. Herein, we collected three different chemotypes of $C$. burmannii, which harbor different contents of D-borneol. A total of 100,218 unigenes with an N50 of 1,128 bp were assembled de novo using Trinity from a total of $21.21 \mathrm{~Gb}$ clean bases. We used BLASTx analysis against several public databases to annotate 45,485 unigenes (45.38\%) to at least one database, among which 82 unigenes were assigned to terpenoid biosynthesis pathways by KEGG annotation. In addition, we defined 8,860 unigenes as differentially expressed genes (DEGs), among which 13 DEGs were associated with terpenoid biosynthesis pathways. One 1-deoxy-D-xylulose-5-phosphate synthase (DXS) and two monoterpene synthase unigenes, designated CbDXS9, CbTPS2 and CbTPS3, were up-regulated in the high-borneol group compared to the low-borneol and borneol-free groups, and might be vital to biosynthesis of D-borneol in C. burmannii. In addition, we identified one WRKY, two BHLH, one AP2/ERF and three MYB candidate genes, which exhibited the same expression patterns as CbTPS2 and CbTPS3, suggesting that these transcription factors might potentially regulate D-borneol biosynthesis. Finally,quantitative real-time PCR was conducted to detect the actual expression level of those candidate genes related to the D-borneol biosynthesis pathway, and the result showed that the expression patterns of the candidate genes related to D-borneol biosynthesis were basically consistent with those revealed by transcriptome analysis.

Conclusions: We used transcriptome sequencing to analyze three different chemotypes of $C$. burmannii, identifying three candidate structural genes (one DXS, two monoterpenes) and seven potential transcription factor unigenes (one WRKY, two BHLH, one AP2/ERF and three MYB) involved in D-borneol biosynthesis. These results will provide new insight into our understanding of the production and accumulation of D-borneol in C. burmannii. 
1 Mining of candidate genes involved in the biosynthesis of

2 dextrorotatory borneol in Cinnamomum burmannii by

3 transcriptomic analysis on three chemotypes

4

5 Zerui Yang, Wenli An, Shanshan Liu, Yuying Huang, Chunzhu Xie, Song Huang*, Xiasheng 6 Zheng*

7

8

National Engineering Research Center for Modernization of Traditional Chinese Medicine, Mathematical Engineering Academy of Chinese Medicine, Guangzhou University of Chinese Medicine, Guangzhou, Guangdong, China

Zerui Yang, Email: yzr_gzucm1991@163.com.

Wenli An, Email: 18434376623@163.com.

Shanshan Liu, Email: 1744832706@qq.com.

Yuying Huang, Email: 13202078270@163.com.

Chunzhu Xie, Email: xiechunzhu2016@126.com.

Corresponding Author:

Song Huang

232th Waihuangdong Road, Higher Education Mega Center, Panyu District, Guangzhou, China Email address: huangnn421@163.com.

Phone: +8613668987309 .

Xiasheng Zheng

232th Waihuangdong Road, Higher Education Mega Center, Panyu District, Guangzhou, China

Email address: xszheng@gzucm.edu.cn

Phone: +8618680167020 . 


\section{Abstract}

Background. Dextrorotatory borneol (D-borneol), a cyclic monoterpene, is widely used in traditional Chinese medicine as an efficient topical analgesic drug. Fresh leaves of Cinnamomum trees, e.g. C. burmannii and C. camphor, are the main sources from which D-borneol is extracted by steam distillation, yet with low yields. Insufficient supply of D-borneol has hampered its clinical use and production of patent remedies for a long time. Biological synthesis of D-borneol offers an additional approach; however, mechanisms of D-borneol biosynthesis remain mostly unresolved. Hence, it is important and necessary to elucidate the biosynthetic pathway of Dborneol.

Results. Comparative analysis on the gene expression patterns of different D-borneol production C. burmanni samples facilitates elucidation on the underlying biosynthetic pathway of D-borneol. Herein, we collected three different chemotypes of $C$. burmannii, which harbor different contents of D-borneol. A total of 100,218 unigenes with an N50 of 1,128 bp were assembled de novo using Trinity from a total of $21.21 \mathrm{~Gb}$ clean bases. We used BLASTx analysis against several public databases to annotate 45,485 unigenes (45.38\%) to at least one database, among which 82 unigenes were assigned to terpenoid biosynthesis pathways by KEGG annotation. In addition, we defined 8,860 unigenes as differentially expressed genes (DEGs), among which 13 DEGs were associated with terpenoid biosynthesis pathways. One 1-deoxy-D-xylulose-5-phosphate synthase (DXS) and two monoterpene synthase unigenes, designated CbDXS9, CbTPS2 and CbTPS3, were upregulated in the high-borneol group compared to the low-borneol and borneol-free groups, and might be vital to biosynthesis of D-borneol in C. burmannii. In addition, we identified one WRKY, two BHLH, one AP2/ERF and three MYB candidate genes, which exhibited the same expression patterns as CbTPS2 and CbTPS3, suggesting that these transcription factors might potentially regulate D-borneol biosynthesis. Finally, quantitative real-time PCR was conducted to detect the actual expression level of those candidate genes related to the D-borneol biosynthesis pathway, and the result showed that the expression patterns of the candidate genes related to D-borneol biosynthesis were basically consistent with those revealed by transcriptome analysis.

Conclusions. We used transcriptome sequencing to analyze three different chemotypes of $C$. burmannii, identifying three candidate structural genes (one DXS, two monoterpenes) and seven potential transcription factor unigenes (one WRKY, two BHLH, one AP2/ERF and three MYB) 
57 involved in D-borneol biosynthesis. These results will provide new insight into our understanding 58 of the production and accumulation of D-borneol in C. burmannii.

59 


\section{Introduction}

D-Borneol, also known as Tianran Bingpian in Chinese, is a time-honored drug in traditional Chinese medicine used for disease prevention and treatment for more than 2,000 years (Guogang 2015; Pharmacopoeia 2015; Shizhen 2004). D-Borneol can be administered either transdermally or orally, with the former most frequently used. Transdermal administration is mainly used to ease pain resulting from wounds, injuries, burns, cuts and similar. When administered orally, D-borneol is widely used to treat cardiovascular diseases, including stroke, coronary heart disease and angina pectoris as an indispensable ingredient in many traditional Chinese herbal formulas, such as Angong Niuhuang pill, Suxiao Jiuxin pill and Fufang Danshen pill (or Compound Danshen) (Chai et al. 2019; Chen et al. 2019; Huang et al. 2016; Liang et al. 2018; Ren et al. 2018) .

Natural sources of D-borneol include the resin and essential oils of several plant families, including Dipterocarpaceae, Lamiaceae, Valerianaceae and Asteraceae. Among these, the Cinnamomum genus within Lamiaceae contributes the majority of D-borneol. C. burmannii harbors D-borneol (19.68\%-78.6\%) as the major component of its essential oil (Chen 2011; Li Y 1987). Steam distillation of $C$. burmannii leaves is the current approach for obtaining D-borneol. However, this traditional extraction method is costly and consumes a great amount of labor and energy. Moreover, it is difficult to achieve sustainable industrial production due to limited natural resources. Synthetic borneol, which is chemically synthesized from turpentine oil or camphor, is essentially impure, containing unexpected byproducts such as levogyral borneol and optically inactive isoborneol. In addition to those byproducts, residual raw material from the chemical reaction producing borneol, e.g. camphor, appears to be harmful to the human body (Cheng et al. 2013; Mathen et al. 2018; Nchinech et al. 2019; Yang et al. 2018). Furthermore, it has been reported that natural D-borneol exhibits higher efficacy than synthetic borneol (Zou et al. 2017). Therefore, the application of synthetic borneol has been restricted. In light of this, it is important to provide a new and sustainable source, e.g. heterologous reconstitution of biosynthesis, for the production of D-borneol.

D-Borneol is a bicyclic monoterpene, one of the members of the terpene family. The metabolic pathways of volatile terpenes have been well characterized in the plant kingdom (Alquezar et al. 2017; Bohlmann et al. 1998). Generally, these terpenoids are all derived from the common precursors isopentyl diphosphate (IPP) and dimethylallyl diphosphate (DMAPP). IPP is biosynthesized in plants by the mevalonate pathway in the cytoplasm or through the methylerythritol phosphate pathway in the plastid by a multi-step reaction starting from acetylCoA or oleic acid, respectively. IPP can then be converted into DMAPP by the catalysis of isopentyl pyrophosphate isomerase (IPPI) (Stage 1, Fig. 1a). IPP and DMAPP are further catalyzed by geranyl diphosphate synthase (GPPS), geranyl geranyl diphosphate synthase (GGPPS) or farnesyl diphosphate synthase (FPPS) to form geranyl diphosphate (GPP), geranyl geranyl diphosphate (GGPP) or farnesyl diphosphate (FPP) (Stage 2, Fig. 1a). These prenyl diphosphates are subsequently catalyzed into mono-, sesqui- or diterpenes by terpene synthase (TPS) (Stage 3, Fig. 1a). The biosynthesis pathway of D-borneol has been partially elucidated in previous studies. Firstly, GPP is converted to bornyl pyrophosphase (BPP) by the catalysis of bornyl pyrophosphase 
synthase (BPPS). BPP is then dephosphorylated to form borneol (Fig. 1b) (Croteau 1998; Despinasse et al. 2017; Hurd et al. 2017; Wang et al. 2018). The BPPS gene has been functionally characterized in four species, Salvia officinalis, Lavandula angustifolia, Lippia dulcis and Amomum villosum. Nevertheless, these four species are not rich in D-borneol, containing only $0.98 \%, 22.63 \%, 1.12 \%$ and $2.87 \%$ D-borneol in their essential oils, respectively. Therefore, BPPS enzymes isolated from these species are of low catalytic efficiency. C. burmannii contains much more D-borneol than any of the above-mentioned species, and thus represents an ideal material for mining potential genes related to D-borneol biosynthesis with high catalytic efficiency (Cardia et al. 2018; Compadre et al. 1986; Koubaa et al. 2019). Nevertheless, to the best of our knowledge, genes which are responsible for the D-borneol biosynthesis in C. burmannii still remain unknown due to the lack of genomic and transcriptomic data.

Transcriptome studies provide a useful perspective for expounding the molecular mechanisms of gene functions, cellular reactions and different biological processes (Kashyap et al. 2020; Quintana-Escobar et al. 2019). To date, many transcriptome analyses aimed at elucidating molecular mechanisms related to biosynthesis of major terpenoids have been performed in Cinnamomum plants, such as Cinnamomum camphora (Chen et al. 2018) and Cinnamomum porrectum (Qiu et al. 2019). Genes with specific expression or higher expression in one of several chemotypes have been identified, laying the foundation for subsequent identification of their corresponding functions. Systematic analysis of genes involved in D-borneol biosynthesis through high-throughput sequencing in C. burmannii will significantly aid mining of a high-efficiency enzyme source for the construction of microbially engineered bacteria with high yields of Dborneol. Here, we compared the transcriptomes of $C$. burmannii chemotypes with three different D-borneol contents by high-throughput sequencing to seek candidate genes related to D-borneol biosynthesis.

\section{Materials and Methods}

\section{Plant material, chemicals and reagents}

Ten-year-old Cinnamomum burmannii of low-content borneol type (LBT, $0<$ D-borneol $<1 \mathrm{mg} / \mathrm{g}$ ) and borneol-free type (FBT) were planted in the Medicinal Botanical Garden of Guangzhou University of Chinese Medicine. Samples of ten-year-old C. burmannii of high-content borneol type (HBT; D-borneol >= $1 \mathrm{mg} / \mathrm{g}$ ) samples were donated by Guangdong Huaqingyuan Biotechnology Co. LTD. Authentic standard D-borneol (purity $>98 \%$ ) was purchased from Shanghai Yuanye Bio-Technology Co., Ltd.

\section{Gas chromatograohy-mass spectrometry (GC/MS) analysis of chemical contents}

Leaves collected from different individual trees of $C$. burmannii were ground into fine powder in liquid nitrogen. Powder samples (0.05-0.10 g) were weighed accurately, soaked in $2.0 \mathrm{~mL}$ petroleum ether using an ultrasonic cleaner for $30 \mathrm{~min}$, and centrifuged at 16,000 rpm for $5 \mathrm{~min}$ in preparation for GC/MS analysis. Three technical replicates were performed for each of the three biological samples. An Agilent 7890B Gas Chromatograph with 5977A Inert Mass Selective 
Detector (Agilent, USA) was used to analyze the extraction. Helium was used as the carrier gas with a flow rate of $1 \mathrm{~mL} / \mathrm{min}$. A Cyclosil-B GC column $(30 \mathrm{~m} \times 0.25 \mathrm{~mm} \times 0.25 \mu \mathrm{m})$ with initial temperature of $50{ }^{\circ} \mathrm{C}$ was used to separate samples. The $\mathrm{GC}$ column temperature program was as follows: oven temperature was increased from $50{ }^{\circ} \mathrm{C}$ to $180{ }^{\circ} \mathrm{C}$ at $2{ }^{\circ} \mathrm{C} / \mathrm{min}$, and then from 180 ${ }^{\circ} \mathrm{C}$ to $300{ }^{\circ} \mathrm{C}$ at $4{ }^{\circ} \mathrm{C} / \mathrm{min}$, then held at $300{ }^{\circ} \mathrm{C}$ for $4 \mathrm{~min}$. Injector: split mode with split ratio of 20:1, injection volume of $1.0 \mu \mathrm{L}$ and inlet temperature of $250^{\circ} \mathrm{C}$. D-Borneol was confirmed by comparing its retention time with that of the known standard, which was determined under the same conditions. The concentration of D-borneol in the plant sample was calculated from its respective standard curves.

\section{RNA extraction, cDNA library preparation and sequencing}

Total RNA of samples collected form the three groups was extracted using TRizol reagent according to the manufacturer's instructions and then digested with RNase-free DNase to eliminate the contamination of genomic DNA. The integrity and quantity of the RNA samples were analyzed using 1\% agarose gel electrophoresis and with a NanoDrop 2000 Spectrophotometer (Thermo Scientific, USA). Qualified RNA samples $\left(\mathrm{OD}_{260} / 280=1.8 \sim 2.2, \mathrm{OD}_{260} / 230 \geq 2.0, \mathrm{RIN} \geq 6.5\right.$, $28 \mathrm{~S}: 18 \mathrm{~S} \geq 1.0$ ) were immediately frozen at $-80{ }^{\circ} \mathrm{C}$ until use. To analyze the transcriptome, $2 \mu \mathrm{g}$ qualified RNA samples from three biological replicates were mixed and sent to Majorbio (Shanghai, China) to construct a cDNA library on an Illumina Hiseq X Ten (Illumina Inc., San Diego, CA, USA). Paired-end (PE) reads were generated and checked by the software fastx_toolkit_0.0.14 (http://hannonlab.cshl.edu/fastx_toolkit/) to assess the quality of sequences.

\section{De novo Transcriptome Assembly and Annotation}

High-quality sequencing data was de novo assembled using TRINITY v2.5.0 (https://github.com/trinityrnaseq/trinityrnaseq) with default parameters, which integrates three independent software modules (Inchworm, Chrysalis and Butterfly) to process and splice a large amount of RNA-Seq data in turn. First, the Inchworm software was used to assemble the clean data into linear contigs. Second, the overlapping contigs were clustered into sets of connected components using the Chrysalis software. Finally, transcripts were constructed using the Butterfly software. The assembly results were further filtered and optimized using TransRate (http://hibberdlab.com/transrate/) and clustered to obtain non-redundant unigenes using CD-HIT (http://weizhongli-lab.org/cd-hit/). For gene identification and expression analysis, the reads from different chemotypes were co-assembled, and for gene sequence analysis, reads from different chemotypes were assembled separately.

Annotation of unigenes was performed using the BLAST program with an E-value cut-off of 1E-5 against the following three databases: NCBI non-redundant protein sequences $(\mathrm{Nr}$, https://blast.ncbi.nlm.nih.gov/), Protein family (Pfam, https://pfam.sanger.ac.uk/) and Swiss-Prot (https://web.expasy.org/docs/swiss-protguideline. html). Functional annotation using gene ontology (GO) terms was analyzed using Blast2GO version 2.5.0 (geneontology.org). Kyoto 
encyclopedia of genes and genomes (KEGG) pathways analysis was performed using KOBAS version 2.1.1 (https://www.genome.jp/kegg/) for systematic analysis of gene function.

\section{Differential gene expression analysis}

Fragments Per Kilobases per Million-reads (FPKM) was used to estimate relative expression levels of transcripts using the software RSEM v1.2.15 (http://deweylab.github.io/RSEM/) with default parameters. Raw counts were directly analyzed statistically using DESeq2 version 1.10 .1 software based on the negative binomial distribution. Unigenes with expression differences among the groups were obtained with the parameters p-adjust $<0.05$ and $|\log 2 \mathrm{FC}| \geq 1$. KEGG enrichment analysis of differentially expressed genes (DEGs) was performed using KOBAS version 2.1.1 (http://www.genome.jp/kegg/).

\section{Screening of candidate TPS genes and transcription factors related to D-borneol biosynthesis}

Candidate TPS genes were first identified by their KEGG annotation and selected by their length and RPKM expression value. Up-regulated genes were then selected for further analysis. The coding sequences of the candidate TPS genes were translated into amino acid sequences, and a phylogenetic tree was reconstructed from the aligned sequences using the neighbor-joining algorithm in MEGA6.0 (Tamura et al. 2013) with 1,000 bootstrap replicates. Transcription factors were analyzed using the HMMER method (Thiriet-Rupert et al. 2016) and their sequences aligned using the plantTFDB (http://planttfdb.cbi.pku.edu.cn/); they were divided into different families according to their conserved domains.

\section{Validation of DEGs by qRT-PCR analysis}

Quantitative reverse-transcription polymerase chain reaction (qRT-PCR) was conducted using TransStar Tip Green qPCR SuperMix (TransGen Biotech, Beijing, China) and the CFX96 Touch Deep Well platform (Bio-Rad, USA) with a total reaction volume of $20 \mu \mathrm{L}$, comprising $1 \mu \mathrm{L}$ cDNA, $10 \mu \mathrm{L}$ Tip Green qPCR Supermix, $0.4 \mu \mathrm{L} 10 \mu \mathrm{M}$ each primer and $8.2 \mu \mathrm{L}$ nuclease-free water. Nine differentially expressed unigenes related to monoterpenenoid biosynthesis and six candidate transcription factors related to D-borneol accumulation were selected, including CbTPS2 (c122670.graph_c0) and CbTPS3 (c129067.graph_c0), CbGPPS_5 (c117157.graph_c0), CbDXR (c84883.graph_c0), CbDXS_1 (c126058. graph_c0), CbDXS_3 (c134634.graph_c0), CbHDS_1 (c134509.graph_c0), CbHDR (c121089.graph_c0), CbHMGR_1 (c133256.graph_c0), CbAP18/ERF-1 (c130684.graph_c1), CbbHLH-1 (c117041.graph_c0), CbbHLH-2 (c127743.graph_c0),
CbMYB-1
(c120253.graph_c0),
CbMYB-2 (c123637.graph_c0), CbWRKY-1 (c122191.graph_c0). The $\beta$-tubulin gene (c121493.graph_c0) was used as a reference gene. Primer premier 5 software (Premier Biosoft Intl., CA USA) was used to design primers for the selected unigenes; coding sequences and primers are listed in Supplementary data 1-2. The qRT-PCR procedure was as follows: $94{ }^{\circ} \mathrm{C}$ for $5 \mathrm{~min}, 40-45$ cycles of $94{ }^{\circ} \mathrm{C}$ for $5 \mathrm{~s}, 60^{\circ} \mathrm{C}$ for $15 \mathrm{~s}$ and $72{ }^{\circ} \mathrm{C}$ for $10 \mathrm{~s}$, followed by a dissociation stage. Expression 
219

220

221

222

223

224

225

226

227

228

229

230

231

232

233

234

235

236

237

238

239

240

241

242

243

244

245

246

247

248

249

250

251

252

253

254

255

256

levels were evaluated using the $2^{-\Delta \Delta \mathrm{Ct}}$ method, and the $\mathrm{Ct}$ values for all genes were normalized to the $\mathrm{Ct}$ value of $\beta$-tubulin.

\section{Results}

\section{D-borneol quantitative determination in three chemotypes of $C$. burmanni}

In previous work, we collected three different chemotypes (HBT, LBT, BFT) of C. burmannii exhibiting similar morphology (Fig. 2a-c). These chemotypes possessed high, low and absent levels of D-borneol, respectively. Here, we used a GC/MS method to analyze the composition of volatile oil in these C. burmannii samples (Fig. 2d-j). A total of 28 compounds were detected in the three chemotype samples, of which nine were common to all three. D-borneol was the most abundant component of the volatile terpenoids in both HBT and LBT groups, accounting for $42.23 \%$ and $57.26 \%$, respectively. In the BFT group, the main component was cymene, accounting for $27.44 \%$ of the volatile terpenoids (Fig. 2k-n, Supplementary data 3-4).

\section{Transcriptome analysis}

To identify genes related to the biosynthesis of D-borneol, we performed deep transcriptome sequencing of the three C. burmanni chemotypes using an Illumina HiSeq 2000 platform. In total, $21.21 \mathrm{~Gb}$ of clean sequence data with $\mathrm{GC}$ content of $47.55 \%$ was obtained from all samples after filtering and removing adapter sequences from the raw data: $8.40 \mathrm{~Gb}$ from the HBT group, 6.39 $\mathrm{Gb}$ from the LBT group and $6.42 \mathrm{~Gb}$ from the BFT group. Raw data and quality control data statistics are summarized in Table 1. All RNA-Seq raw data has been submitted to CNGBdb with accession number CNP0000810; https://db.cngb.org/search/?q= CNP0000810.

For further analysis, high-quality clean reads were assembled using the TRINITY program, resulting in 142,673 transcripts and 100,218 unigenes with N50 of 1,625 bp and 1,128 bp and average length of $935 \mathrm{bp}$ and $713 \mathrm{bp}$, respectively. The majority of the reads for both transcripts and unigenes were shorter than $1,000 \mathrm{bp}$ in length, accounting for $92 \%$ and $88 \%$, respectively. Only $2 \%$ of the transcripts and $3 \%$ of the unigenes were longer than 2,000 bp in length. All assembled data are summarized in Table 2 and Fig. 3a. Moreover, $80.80 \%$ of the clean reads were perfectly mapped to the assembled unigenes by RSEM, indicating that these mapped genes were of high quality and could be used to conduct the subsequent analysis (Table 1). Sequence of the 100,218 unigenes were shown in Supplementary data 5.

All resulting 100,218 unigenes were used for BLAST searches against the GO, KEGG, NR, Pfam and Swiss-Prot databases for annotation, resulting in 28,798, 15,376, 44,495, 28,107 and 24,238 annotated unigenes for the above database, respectively (Fig. 3b, Supplementary data 6). In total, 45,485 unigenes were annotated to at least one database, and 9,730 unigenes shared annotation in all five databases (Fig. 3c). The species distribution of the annotated unigenes is shown in Fig. 3d.

\section{Identification of DEGs}


257

258

259

260

261

262

263

264

265

266

267

268

269

A total of 8,860 DEGs were identified among the comparison groups. The expression patterns of these DEGs could be classified into four clusters (Fig. 3e-f, Supplementary data 7). Cluster 1 consisted of 2,600 genes significantly down-regulated in the LBT group in comparison with both the HBT and BFT groups. Cluster 2 exhibited the opposite expression pattern to that of cluster 1 , containing 3,721 unigenes that were particularly up-regulated in the LBT group in comparison to both the HBT and BFT groups. Cluster 3 contained 2,539 unigenes significantly up-regulated in the BFT group in comparison to both the HBT and LBT groups. Ten genes significantly downregulated in the BFT group in comparison to both the HBT and LBT groups formed cluster 4. We used a Wayne diagram (Fig. 3g) to show the number of DEGs between each group. There were 3,140 up-regulated unigenes and 3,643 down-regulated unigenes in the HBT vs LBT comparison. In the HBT vs BFT comparison, we identified 1,303 up-regulated unigenes and 924 downregulated unigenes. In the LBT vs BFT comparison, 3,902 unigenes were up-regulated while 2,357 unigenes were down-regulated (Fig. 3h).

To gain a deep understanding of the biological functions of these 8,860 DEGs, we performed KEGG pathway enrichment analysis. We used dot plots to show the top 20 most enriched KEGG pathway categories with $p$ value $<0.05$, which indicated that the top three most enriched KEGG pathways were "cutin, suberin and wax biosynthesis," "vancomycin resistance" and "fatty acid degradation" (Fig. 4a, Supplementary data 8). The top 10 most enriched GO terms under the three general sections Biological Process (BP), Cellular Component (CC) and Molecular Function (MF) are summarized in Fig. 4b and Supplementary data 9. The most enriched GO term in the MF category was protein serine/threonine kinase activity. Within the CC category, the most abundant GO term was chloroplast. In the BP category, the most associated term was protein phosphorylation. In addition, terpene synthase activity was also significantly enriched.

\section{Mining of candidate genes related to D-borneol biosynthesis}

Monoterpenes derived from plants are biosynthesized in the plastid through the MEP pathway. We detected 30 unigenes involved in the MEP pathway, but only three of them exhibited significantly different expression levels among the three comparison groups. Most of the unigenes (11/30), including one DEG, were annotated as $D X S$ genes, which encode the first rate-limiting enzyme in the MEP pathway. Relatively higher expression of the $D X S$ gene in the HBT group compared to that in the other two groups might contribute to the differences in D-borneol accumulation in $C$. burmannii. Six unigenes were annotated as encoding GPPS, responsible for GPP formation. It is well known that GPPSs exist as homomeric and heteromeric structures in plants (Burke et al. 2004; Rai et al. 2013; Wang \& Dixon 2009; Yin et al. 2017) and that plants contain multiple GGPPS or GGPPS-related enzymes, some functioning as large or small subunits (LSU/SSU) of heteromeric GPPS (Tholl et al. 2004; Wang \& Dixon 2009). For example, there are 12 GGPPS paralogs in the Arabidopsis genome(Beck et al. 2013; Lange \& Ghassemian 2003). For example, there are 12 GGPPS paralogs in the Arabidopsis genome (Beck et al. 2013; Lange \& Ghassemian 2003); however, AtGPPS11 and AtGGPPS12 were characterized as LSU and SSU, with their interaction involved in monoterpene biosynthesis in Arabidopsis flowers (Chen et al. 2015). To confirm 
whether the $C$. burmannii GPPSs formed homomeric or heteromeric structures, we conducted a phylogenetic analysis of seven KEGG-annotated CbGPPS/GGPPS proteins from C. burmannii and the amino acid sequences of other characterized GPPS/GGPPS proteins. We identified six GPPSs, including two putative homomeric GPPSs, three putative GPPS.SSUs and a putative GPPS.LSU. Only one unigene may act as a GGPPS (Fig.S1). It has been reported that monoterpene biosynthesis in gymnosperms is mainly regulated by homomeric GPPS and SSUs of heteromeric GPPS (Schmidt et al. 2010; Tholl et al. 2004). The 2 putative homomeric GPPSs, 3 putative GPPS.SSUs identified in this study may provide a possible explanation for the monoterpene richness of C. burmanni.

There were 40 unigenes annotated as TPS by KEGG, among which 11 were associated with the sesquiterpenoid and triterpenoid biosynthesis pathway (ko00904), eight were monoterpenoid synthase unigenes (ko00902) and 21 encoded diterpenoid synthase (ko00909). Since D-borneol is a monoterpene, the eight putative monoterpenoid synthase genes were used for further analysis. The expression patterns of the eight unigenes encoding monoterpenoid synthase, along with those of genes related to the MEP metabolic pathway (Ko00900), were normalized and are shown in Fig. 5a-b and Supplementary data 10. Considering the relatively high accumulation of Dborneol in the HBT group compared to the other two groups, we rationalized that metabolismrelated genes might exhibit more activity in the HBT group than in the other two groups. Inspiringly, two unigenes encoding monoterpenoid synthase (designated CbTPS2 and CbTPS3) were significantly up-regulated in the HBT group compared with the LBT and BFT groups (Supplementary data 11). Furthermore, both unigenes had complete open reading frames. Sequence comparison using BLAST indicated that CbTPS2 (c122670.graph_c0) exhibited highest similarity [53.27\% amino acid (aa) sequence identity] to an (E)-beta-ocimene/myrcene synthase (ADR74206.1) from Vitis vinifera, while CbTPS3 (c129067.graph_c0) presented similarity in aa sequence with a S-(+)-linalool synthase (RVW86370.1) from Vitis vinifera (Martin et al. 2010).

These two candidate TPS genes were subjected to a phylogenetic analysis, along with other $T P S$ genes that have been functionally characterized. Similar to other well-characterized BPPS genes, CbTPS2 fell within the TPS-b subfamily (Fig. S2). Furthermore, sequence alignment showed that CbTPS2 had conserved mono-TPS domains, including RRX8W, RXR, DDXXD and (N,D) D (L,I,V) X (S,T) XXE (NSE/DTE) (Fig. S3). To find out whether the candidate unigenes differed between each chemotype, the transcriptomes of the three groups were assembled separately, and the sequences were compared. The assembled sequences of each chemotypes were shown in Supplementary data 12-14. The amino acid sequences of CbTPS2 in the three different C. burmannii chemotypes differed only by two amino acids, and these sites were not located within the conserved domain, suggesting that these differences may not affect the function of these proteins (Fig. S4). However, it is interesting that the complete sequence of CbTPS3 could only be found in the HBT and LBT groups, whereas only a small fragment was found in the BFT group (Fig. S5). This may be due to poor assembly of the BFT transcriptome, or perhaps CbTPS3 is a pseudogene in BFT. We speculated that CbTPS2 and CbTPS3 are probably candidate genes responsible for the biosynthesis of D-borneol in C. burmannii. 
Mining of candidate transcription factors related to D-borneol accumulation

Transcription factors play an important role in regulating the biosynthesis and accumulation of secondary metabolites (Hong et al. 2012). In our transcriptome analysis, we identified 1,108 transcription factors, which could be assigned to 47 families (Supplementary data 15). Several TF families, including MYB, basic helix-loop-helix (bHLH), WRKY, basic region/leucine zipper motif (bZIP) and APETALA2/ethylene-response factor (AP2/ERF) families, function in regulating the structural genes involved in monoterpenoid biosynthesis (SU Wen-Bing 2019) . These five TF families cover most of the TFs identified in this study (Fig. 5c). We identified 116 MYB, 65 AP2/ERF, 63 BHLH, 60 bZIP and 47 WRKY candidate genes, among which 35 MYB, 27 AP2/ERF, 24 BHLH, 10 bZIP and 9 WRKY genes were classified as DEGs (Fig. 5d-h, Supplementary data 16). Notably, one WRKY (c122191.graph.c0), two BHLH (c117041.graph.c0, c127743.graph.c0), one AP2/ERF (c130684.graph.c1) and three MYB candidate genes (c68268.graph.c0, c120253. graph.c0, c123637.graph.c0) were significantly upregulated in both the HBT and LBT groups compared to the BFT group. The different transcription factor expression patterns might be responsible for regulating monoterpenoid biosynthesis. Moreover, the up-regulated transcription factors in the HBT and LBT groups compared to the BFT group provide us with a candidate TF pool related to the biosynthesis and accumulation of Dborneol.

\section{Validation of DEGs using Quantitative real-time PCR (qRT-PCR)}

To verify the accuracy of RNA-Seq data, we performed expression level analysis on nine unigenes related to the terpenoid biosynthesis pathway and six candidate transcription factors related to Dborneol accumulation using the qRT-PCR method. As shown in Fig. 6, their expression patterns were similar to those revealed from RNA-Seq data. Thus, these results help confirm the reliability of the transcriptomic analysis.

\section{Discussion}

D-Borneol is an effective topical pain reliever for humans, proven in clinical studies (Wang et al. 2017). It is therefore attracting more and more attention from researchers. In China, current Dborneol production is from fresh leaves of $C$. burmannii by water distillation. However, balancing the supply and demand of D-borneol is a challenge due to the long plant growth period, unsatisfactorily low yield and the huge consumption of labor and energy. Authenticating highefficiency enzymes associated with the biosynthesis of D-borneol will benefit heterologous recombination, which could help significantly in solving the supply problem. However, deficiency of $C$. burmannii genomic and transcriptomic information increases the difficulty of identifying core enzymes associated with D-borneol biosynthesis. Our transcriptome analysis of C. burmannii may help to uncover genes related to the biosynthesis of D-borneol through bioinformatics analysis.

It is a common phenomenon that noteworthy differences in chemical composition exist between individuals of the same species in the genus Cinnamomum (Guo et al. 2017; Singh et al. 
2012; Teles et al. 2019). For example, there are at least five chemotypes in C. camphora, including the borneol type, linalool type, camphor type, nerolidol type and 1,8-cineole type (Chen et al. 2018). Cinnamomum tenuipilum harbors six chemotypes, including the methyleugenol type, citral type, linalool type, geraniol type, camphor type and farnesol type (Xuefen 1991). In C. burmannii, we also found great differences in the content of D-borneol in different individuals, despite it being reported to be a D-borneol rich species. The molecular mechanism of chemotype formation in Cinnamomum has not yet been elucidated, but studies on other plants such as Melaleuca alternifolia, which is rich in terpenes and has different chemotypes, indicates that a difference in TPS may be responsible for the phenomenon of multiple chemotypes within the species (BustosSegura et al. 2017; Padovan et al. 2017).

Transcriptome analysis is currently the most widely used and cost effective way to screen target gene(s) rapidly in functional genomic studies (Ali et al. 2018; Ma et al. 2019; Zhou et al. 2019). In this study, a total of 100,218 unigenes were generated by transtomic sequencing of three chemotypes of $C$. burmannii. In detail, 45,485 unigenes (45.38\%) succeeded in functional annotation against several public databases. However, the annotation rate of unigene assembled in this study appear to be quite low, compared to other species, which may be due to the variable splicing of transcripts and the deficient of genomic sequences (Liang et al. 2019).

In the past few decades, genes involved in the biosynthesis of terpenes have been verified in many plants (Abbas et al. 2019; Anand et al. 2019; Vishal et al. 2019). However, although species within the Cinnamomum genus are rich in terpenes, only one gene encoding geraniol synthase has been isolated and functionally characterized from C. tenuipilum (Yang et al. 2005). Transcriptome study of different chemotypes of $C$. camphora indicated that 67 genes might be involved in terpene biosynthesis, of which three genes (CcTPS14-like1, CcTPS14-like2, CcTPS14-like3) were significantly upregulated in the borneol chemotype relative to the linalool chemotype. These three genes may play an important role in terpenoid accumulation in the borneol-type of C. camphora (Chen et al. 2018). Transcriptome analysis of three chemotypes of C. porrectum identified 52, 49 and 66 candidate unigenes related to terpene biosynthesis in the eucalyptol chemotype and linalool chemotype, the camphor chemotype and eucalyptol chemotype, and the camphor chemotype and linalool chemotype comparison groups, respectively. The terpenoid synthase genes CpTPS1, CPTPS3, CPTPS4, CPTPS5 and CpTPS9 had specific expression or higher expression in one of the chemotypes and may be involved in monoterpene biosynthesis (Qiu et al. 2019). The transcriptome and bioinformatics analysis performed in this study aimed to uncover candidate genes related to the biosynthesis of D-borneol. Compared to the published Cinnamomum transcriptome, we found more genes related to terpenoid biosynthesis, but fewer genes related to specific terpenoid biosynthesis. We annotated a total of 82 genes as related to terpene biosynthesis, 52 of which participated in the biosynthesis of the terpene backbone and 30 of which were annotated as TPS (Supplementary data 17). Only 13 of these 94 genes were DEGs, including one $D X S$ gene and two genes encoding monoterpene synthase. DXS is regarded as the first rate-limiting enzyme in the MEP pathway, which provides corresponding precursors for monoterpene and diterpene. Of the two monoterpene synthases identified in this study, CbTPS3 was annotated as a 
TPS14-like gene, which mediates the biosynthesis of linalool as reported in Arabidopsis thaliana (Chen et al. 2003). Interestingly, although CbTPS3 was actively expressed, no or little linalool was found in C. burmannii. This phenomenon was also observed in the closely related species $C$. camphora (Chen et al. 2018). Furthermore, we found that CbTPS3 probably only exists in the borneol chemotype of $C$. burmannii. Therefore, whether CbTPS3 is involved in the biosynthesis of D-borneol needs further examination. The other monoterpene synthase gene, CbTPS2, was clustered into the TPSb subfamily. Nevertheless, functional authentication remains to be verified in a future study, regarding to the low homology among those reported known TPSb-subfamily genes which have the same catalytic function (Hosoi et al. 2004; Landmann et al. 2007).

Transcription factors activate synergistic expression of multiple genes encoding secondary metabolite synthases, thereby effectively regulating secondary metabolic pathways. Plant terpenoid biosynthesis is mainly regulated by transcription factors, such as bZIP, WRKY, bHLH, AP2/ERF and MYB family members. For example, a PGT-specific R2R3-MYB gene, MsMYB, from Mentha spicata regulates monoterpene production and suppresses the expression of the geranyl diphosphate synthase large subunit (Reddy et al. 2017). Transient ectopic expression of bZIP TF genes isolated from Phalaenopsis bellina, designated PbbZIP, induces a 10-fold increase in monoterpenoid production in the scentless orchid (Chuang et al. 2018). A jasmonate-responsive bHLH factor participates in modulating monoterpenoid indole alkaloid biosynthesis in Catharanthus roseus (Patra et al. 2018). Transcription factor CitERF71 activates the terpene synthase encoding gene CitTPS16 involved in the biosynthesis of E-geraniol in Citrus sinensis Osbeck (Li et al. 2017). Recently, a genome-wide investigation of WRKY transcription factors in Osmanthus Fragrans indicated potential roles for OfWRKY139 and OfWRKYs with plant zinc cluster domains in regulating biosynthesis of aromatic compounds in sweet osmanthus (Ding et al. 2019); A study on monoterpene synthase promoter related transcription factors in Lavandula $\times$ Intermedia by yeast one-hybrid assay indicated that multiple transcription factors control monoterpene synthase expression in lavender, including MYB, bZIP, NAC, GeBP and SBPrelated proteins (Sarker et al. 2019). In the present work, we identified one WRKY, two BHLH, one $A P 2 / E R F$ and three $M Y B$ candidate genes, which exhibited the same expression pattern as CCTPS5 and CcTPS7. Based on the idea that genes co-expressed under multiple conditions tend to be functionally related (Aoki et al. 2007; Ihmels et al. 2004), elucidating the function of these transcription factors in D-borneol regulation will be the focus of follow-up research.

\section{Conclusion}

To the best of our knowledge, this is the first transcriptomic study in C. burmannii, aimed at discovering genes participating in the biosynthesis of D-borneol. A total of 100,218 unigenes were assembled from our transcriptome sequencing of $C$. burmannii with three different levels of borneol, among which 8,860 unigenes were defined as DEGs. Two of these DEGs, CbTPS2 and CbTPS3, had relatively high expression levels in the C. burmannii chemotype with high D-borneol content. We conclude that these two genes might be directly responsible for the biosynthesis of Dborneol. Furthermore, seven TF genes (one WRKY, two BHLH, one AP2/ERF and three MYB) 
possessing the same expression pattern as CbTPS2 and CbTPS3 might play an important role in regulating D-borneol biosynthesis. However, their specific functions need to be elucidated in further studies. The extensive gene assembly obtained from our transcriptomic sequencing provides a pool of candidate genes for analyzing the accumulation mechanism of D-borneol in $C$. burmannii.

\section{Acknowledgements no}

\section{References}

Abbas F, Ke Y, Yu R, and Fan Y. 2019. Functional characterization and expression analysis of two terpene synthases involved in floral scent formation in Lilium 'Siberia'. Planta 249:71-93. 10.1007/s00425-0183006-7

Ali M, Hussain RM, Rehman NU, She G, Li P, Wan X, Guo L, and Zhao J. 2018. De novo transcriptome sequencing and metabolite profiling analyses reveal the complex metabolic genes involved in the terpenoid biosynthesis in Blue Anise Sage (Salvia guaranitica L.). DNA Res 25:597-617. 10.1093/dnares/dsy028

Alquezar B, Rodriguez A, de la Pena M, and Pena L. 2017. Genomic Analysis of Terpene Synthase Family and Functional Characterization of Seven Sesquiterpene Synthases from Citrus sinensis. Front Plant Sci 8:1481. 10.3389/fpls.2017.01481

Anand A, Jayaramaiah RH, Beedkar SD, Dholakia BB, Lavhale SG, Punekar SA, Gade WN, Thulasiram HV, and Giri AP. 2019. Terpene profiling, transcriptome analysis and characterization of cis-betaterpineol synthase from Ocimum. Physiol Mol Biol Plants 25:47-57. 10.1007/s12298-018-0612-6

Aoki K, Ogata Y, and Shibata D. 2007. Approaches for extracting practical information from gene co-expression networks in plant biology. Plant Cell Physiol 48:381-390. 10.1093/pcp/pcm013

Beck G, Coman D, Herren E, Ruiz-Sola MA, Rodriguez-Concepcion M, Gruissem W, and Vranova E. 2013. Characterization of the GGPP synthase gene family in Arabidopsis thaliana. Plant Mol Biol 82:393-416. 10.1007/s11103-013-0070-z

Bohlmann J, Meyer-Gauen G, and Croteau R. 1998. Plant terpenoid synthases: molecular biology and phylogenetic analysis. Proc Natl Acad Sci U S A 95:4126-4133. 10.1073/pnas.95.8.4126

Burke C, Klettke K, and Croteau R. 2004. Heteromeric geranyl diphosphate synthase from mint: construction of a functional fusion protein and inhibition by bisphosphonate substrate analogs. Arch Biochem Biophys 422:52-60. 10.1016/j.abb.2003.12.003

Bustos-Segura C, Padovan A, Kainer D, Foley WJ, and Kulheim C. 2017. Transcriptome analysis of terpene chemotypes of Melaleuca alternifolia across different tissues. Plant Cell Environ 40:2406-2425. $10.1111 /$ pce. 13048

Cardia GFE, Silva-Filho SE, Silva EL, Uchida NS, Cavalcante HAO, Cassarotti LL, Salvadego VEC, Spironello RA, Bersani-Amado CA, and Cuman RKN. 2018. Effect of Lavender (Lavandula angustifolia) Essential Oil on Acute Inflammatory Response. Evid Based Complement Alternat Med 2018:1413940. 10.1155/2018/1413940

Chai Y, Yin Z, Fan Q, Zhang Z, Ye K, Xu Y, Xiao W, Chai X, Zhu T, and Nie H. 2019. Protective Effects of Angong Niuhuang Pill on Early Atherosclerosis in ApoE(-/-) Mice by Reducing the Inflammatory Response. Evid Based Complement Alternat Med 2019:9747212. 10.1155/2019/9747212

Chen C, Zheng Y, Zhong Y, Wu Y, Li Z, Xu LA, and Xu M. 2018. Transcriptome analysis and identification of genes related to terpenoid biosynthesis in Cinnamomum camphora. BMC Genomics 19:550. 10.1186/s12864-018-4941-1

Chen F, Tholl D, D'Auria JC, Farooq A, Pichersky E, and Gershenzon J. 2003. Biosynthesis and emission of terpenoid volatiles from Arabidopsis flowers. Plant Cell 15:481-494. 10.1105/tpc.007989

Chen LS, Jianyu; Li, Lin; Li, Bing; Li, Wang. 2011. A new source of natural D-borneol and its characteristic. Journal of Medicinal Plants Research 5:7.

Chen Q, Fan D, and Wang G. 2015. Heteromeric Geranyl(geranyl) Diphosphate Synthase Is Involved in Monoterpene Biosynthesis in Arabidopsis Flowers. Mol Plant 8:1434-1437. 10.1016/j.molp.2015.05.001

Chen ZX, Xu QQ, Shan CS, Shi YH, Wang Y, Chang RC, and Zheng GQ. 2019. Borneol for Regulating the Permeability of the Blood-Brain Barrier in Experimental Ischemic Stroke: Preclinical Evidence and Possible Mechanism. Oxid Med Cell Longev 2019:2936737. 10.1155/2019/2936737 
Cheng C, Liu XW, Du FF, Li MJ, Xu F, Wang FQ, Liu Y, Li C, and Sun Y. 2013. Sensitive assay for measurement of volatile borneol, isoborneol, and the metabolite camphor in rat pharmacokinetic study of Borneolum (Bingpian) and Borneolum syntheticum (synthetic Bingpian). Acta Pharmacol Sin 34:13371348. 10.1038/aps.2013.86

Chuang YC, Hung YC, Hsu CY, Yeh CM, Mitsuda N, Ohme-Takagi M, Tsai WC, Chen WH, and Chen HH. 2018. A Dual Repeat Cis-Element Determines Expression of GERANYL DIPHOSPHATE SYNTHASE for Monoterpene Production in Phalaenopsis Orchids. Front Plant Sci 9:765. 10.3389/fpls.2018.00765

Compadre CM, Robbins EF, and Kinghorn AD. 1986. The intensely sweet herb, Lippia dulcis Trev.: historical uses, field inquiries, and constituents. $J$ Ethnopharmacol 15:89-106. 10.1016/0378-8741(86)90105-4

Croteau MLWTJSEKR. 1998. Monoterpene Synthases from Common Sage (Salvia officinalis). THE JOURNAL OF BIOLOGICAL CHEMISTRY 273:10.

Despinasse Y, Fiorucci S, Antonczak S, Moja S, Bony A, Nicole F, Baudino S, Magnard JL, and Jullien F. 2017. Bornyl-diphosphate synthase from Lavandula angustifolia: A major monoterpene synthase involved in essential oil quality. Phytochemistry 137:24-33. 10.1016/j.phytochem.2017.01.015

Ding W, Ouyang Q, Li Y, Shi T, Li L, Yang X, Ji K, Wang L, and Yue Y. 2019. Genome-wide investigation of WRKY transcription factors in sweet osmanthus and their potential regulation role in aroma synthesis. Tree Physiol. 10.1093/treephys/tpz129

Guo X, Cui M, Deng M, Liu X, Huang X, Zhang X, and Luo L. 2017. Molecular differentiation of five Cinnamomum camphora chemotypes using desorption atmospheric pressure chemical ionization mass spectrometry of raw leaves. Sci Rep 7:46579. 10.1038/srep46579

Guogang L. 2015. Luo Shi Hui Yue Yi Jing. Beijing: China Press of Traditional Chinese Medicine.

Hong GJ, Xue XY, Mao YB, Wang LJ, and Chen XY. 2012. Arabidopsis MYC2 interacts with DELLA proteins in regulating sesquiterpene synthase gene expression. Plant Cell 24:2635-2648. 10.1105/tpc.112.098749

Hosoi M, Ito M, Yagura T, Adams RP, and Honda G. 2004. cDNA isolation and functional expression of myrcene synthase from Perilla frutescens. Biol Pharm Bull 27:1979-1985. 10.1248/bpb.27.1979

Huang J, Tang X, Ye F, He J, and Kong X. 2016. Clinical Therapeutic Effects of Aspirin in Combination with Fufang Danshen Diwan, a Traditional Chinese Medicine Formula, on Coronary Heart Disease: A Systematic Review and Meta-Analysis. Cell Physiol Biochem 39:1955-1963. 10.1159/000447892

Hurd MC, Kwon M, and Ro DK. 2017. Functional identification of a Lippia dulcis bornyl diphosphate synthase that contains a duplicated, inhibitory arginine-rich motif. Biochem Biophys Res Commun 490:963-968. 10.1016/j.bbrc.2017.06.147

Ihmels J, Levy R, and Barkai N. 2004. Principles of transcriptional control in the metabolic network of Saccharomyces cerevisiae. Nat Biotechnol 22:86-92. 10.1038/nbt918

Kashyap A, Rhodes A, Kronmiller B, Berger J, Champagne A, Davis EW, Finnegan MV, Geniza M, Hendrix DA, Lohr CV, Petro VM, Sharpton TJ, Wells J, Epps CW, Jaiswal P, Tyler BM, and Ramsey SA. 2020. Pan-tissue transcriptome analysis of long noncoding RNAs in the American beaver Castor canadensis. BMC Genomics 21:153. 10.1186/s12864-019-6432-4

Koubaa FG, Abdennabi R, Salah ASB, and El Feki A. 2019. Microwave extraction of Salvia officinalis essential oil and assessment of its GC-MS identification and protective effects versus vanadium-induced nephrotoxicity in Wistar rats models. Arch Physiol Biochem 125:404-413. $10.1080 / 13813455.2018 .1478427$

Landmann C, Fink B, Festner M, Dregus M, Engel KH, and Schwab W. 2007. Cloning and functional characterization of three terpene synthases from lavender (Lavandula angustifolia). Arch Biochem Biophys 465:417-429. 10.1016/j.abb.2007.06.011

Lange BM, and Ghassemian M. 2003. Genome organization in Arabidopsis thaliana: a survey for genes involved in isoprenoid and chlorophyll metabolism. Plant Mol Biol 51:925-948. 10.1023/a:1023005504702

Li X, Xu Y, Shen S, Yin X, Klee H, Zhang B, Chen K, and Hancock R. 2017. Transcription factor CitERF71 activates the terpene synthase gene CitTPS16 involved in the synthesis of E-geraniol in sweet orange fruit. $J$ Exp Bot 68:4929-4938. 10.1093/jxb/erx316

Li Y ZL, Lu B, Mei L, Li L, Jia L. 1987. Studies of Mei Pian tree (Cinnamomum burmannii physiological type) as a new resource of natural d-borneol. Zhiwu Xuebao 29:5.

Liang CJ, Li JH, Zhang Z, Zhang JY, Liu SQ, and Yang J. 2018. Suppression of MIF-induced neuronal apoptosis may underlie the therapeutic effects of effective components of Fufang Danshen in the treatment of Alzheimer's disease. Acta Pharmacol Sin 39:1421-1438. 10.1038/aps.2017.210 
Liang W, Ni L, Carballar-Lejarazu R, Zou X, Sun W, Wu L, Yuan X, Mao Y, Huang W, and Zou S. 2019. Comparative transcriptome among Euscaphis konishii Hayata tissues and analysis of genes involved in flavonoid biosynthesis and accumulation. BMC Genomics 20:24. 10.1186/s12864-018-5354-x

Ma D, Gao H, Du C, Li L, Sun W, Liu S, Wang C, Xie Y, and Kang G. 2019. Transcriptomic and Metabolomics Analysis of Different Endosperm Region under Nitrogen Treatments. Int J Mol Sci 20. $10.3390 / \mathrm{ijms} 20174212$

Martin DM, Aubourg S, Schouwey MB, Daviet L, Schalk M, Toub O, Lund ST, and BohImann J. 2010. Functional annotation, genome organization and phylogeny of the grapevine (Vitis vinifera) terpene synthase gene family based on genome assembly, FLcDNA cloning, and enzyme assays. BMC Plant Biol 10:226. 10.1186/1471-2229-10-226

Mathen PG, Sreekrishnan TP, Kumar KPG, and Mohan N. 2018. Camphor Poisoning: A Rare Cause of Acute Symptomatic Seizures in Children. J Emerg Trauma Shock 11:228-229. 10.4103/JETS.JETS_21_18

Nchinech N, Elgharbi A, Aglili FZ, Kriouile Y, Cherrah Y, Mdaghri AA, and Serragui S. 2019. [Traditional camphor misuse: a forgotten danger to children (about 2 cases)]. Pan Afr Med J 32:89. 10.11604/pamj.2019.32.89.17943

Padovan A, Keszei A, Hassan Y, Krause ST, Kollner TG, Degenhardt J, Gershenzon J, Kulheim C, and Foley WJ. 2017. Four terpene synthases contribute to the generation of chemotypes in tea tree (Melaleuca alternifolia). BMC Plant Biol 17:160. 10.1186/s12870-017-1107-2

Patra B, Pattanaik S, Schluttenhofer C, and Yuan L. 2018. A network of jasmonate-responsive bHLH factors modulate monoterpenoid indole alkaloid biosynthesis in Catharanthus roseus. New Phytol 217:1566-1581. $10.1111 /$ nph. 14910

Pharmacopoeia. 2015. China pharmacopoeia. Beijing: Committee of National.

Qiu F, Wang X, Zheng Y, Wang H, Liu X, and Su X. 2019. Full-Length Transcriptome Sequencing and Different Chemotype Expression Profile Analysis of Genes Related to Monoterpenoid Biosynthesis in Cinnamomum porrectum. Int J Mol Sci 20. 10.3390/ijms20246230

Quintana-Escobar AO, Nic-Can GI, Galaz Avalos RM, Loyola-Vargas VM, and Gongora-Castillo E. 2019. Transcriptome analysis of the induction of somatic embryogenesis in Coffea canephora and the participation of ARF and Aux/IAA genes. PeerJ 7:e7752. 10.7717/peerj.7752

Rai A, Smita SS, Singh AK, Shanker K, and Nagegowda DA. 2013. Heteromeric and homomeric geranyl diphosphate synthases from Catharanthus roseus and their role in monoterpene indole alkaloid biosynthesis. Mol Plant 6:1531-1549. 10.1093/mp/sst058

Reddy VA, Wang Q, Dhar N, Kumar N, Venkatesh PN, Rajan C, Panicker D, Sridhar V, Mao HZ, and Sarojam R. 2017. Spearmint R2R3-MYB transcription factor MsMYB negatively regulates monoterpene production and suppresses the expression of geranyl diphosphate synthase large subunit (MsGPPS.LSU). Plant Biotechnol J 15:1105-1119. 10.1111/pbi.12701

Ren L, Wang J, Feng L, Wang S, and Li J. 2018. Efficacy of Suxiao Jiuxin Pill on Coronary Heart Disease: A Meta-Analysis of Randomized Controlled Trials. Evid Based Complement Alternat Med 2018:9745804. $10.1155 / 2018 / 9745804$

Sarker LS, Adal AM, and Mahmoud SS. 2019. Diverse transcription factors control monoterpene synthase expression in lavender (Lavandula). Planta 251:5. 10.1007/s00425-019-03298-w

Schmidt A, Wachtler B, Temp U, Krekling T, Seguin A, and Gershenzon J. 2010. A bifunctional geranyl and geranylgeranyl diphosphate synthase is involved in terpene oleoresin formation in Picea abies. Plant Physiol 152:639-655. 10.1104/pp.109.144691

Shizhen L. 2004. Ben Cao Gang Mu. Beijing: Peoples Medical Publishing House.

Singh V, Gupta AK, Singh SP, and Kumar A. 2012. Direct analysis in real time by mass spectrometric technique for determining the variation in metabolite profiles of Cinnamomum tamala Nees and Eberm genotypes. ScientificWorldJournal 2012:549265. 10.1100/2012/549265

SU Wen-Bing JY-Y, BAI Yun-Lu, Gan Xiao-Qing, LIU Yue-Xue, LIN Shun-Quan. 2019. Advances in Transcription Factors Regulation on Plant Terpene Biosynthesis. Journal of Agricultural Biotechnology 27:919.

Tamura K, Stecher G, Peterson D, Filipski A, and Kumar S. 2013. MEGA6: Molecular Evolutionary Genetics Analysis version 6.0. Mol Biol Evol 30:2725-2729. 10.1093/molbev/mst197

Teles AM, Rosa T, Mouchrek AN, Abreu-Silva AL, Calabrese KDS, and Almeida-Souza F. 2019. Cinnamomum zeylanicum, Origanum vulgare, and Curcuma longa Essential Oils: Chemical Composition, Antimicrobial and Antileishmanial Activity. Evid Based Complement Alternat Med 2019:2421695. $10.1155 / 2019 / 2421695$ 
619

620

621

622

623

624

625

626

627

628

629

630

631

632

633

634

635

636

637

638

639

640

641

642

643

644

645

646

647

648

649

650

651

652

653

654

655

Thiriet-Rupert S, Carrier G, Chenais B, Trottier C, Bougaran G, Cadoret JP, Schoefs B, and Saint-Jean B. 2016. Transcription factors in microalgae: genome-wide prediction and comparative analysis. $B M C$ Genomics 17:282. 10.1186/s12864-016-2610-9

Tholl D, Kish CM, Orlova I, Sherman D, Gershenzon J, Pichersky E, and Dudareva N. 2004. Formation of monoterpenes in Antirrhinum majus and Clarkia breweri flowers involves heterodimeric geranyl diphosphate synthases. Plant Cell 16:977-992. 10.1105/tpc.020156

Vishal B, Krishnamurthy P, Ramamoorthy R, and Kumar PP. 2019. OsTPS8 controls yield-related traits and confers salt stress tolerance in rice by enhancing suberin deposition. New Phytol 221:1369-1386. $10.1111 /$ nph. 15464

Wang G, and Dixon RA. 2009. Heterodimeric geranyl(geranyl)diphosphate synthase from hop (Humulus lupulus) and the evolution of monoterpene biosynthesis. Proc Natl Acad Sci U S A 106:9914-9919. 10.1073/pnas.0904069106

Wang H, Ma D, Yang J, Deng K, Li M, Ji X, Zhong L, and Zhao H. 2018. An Integrative Volatile Terpenoid Profiling and Transcriptomics Analysis for Gene Mining and Functional Characterization of AvBPPS and AvPS Involved in the Monoterpenoid Biosynthesis in Amomum villosum. Front Plant Sci 9:846. 10.3389/fpls.2018.00846

Wang S, Zhang D, Hu J, Jia Q, Xu W, Su D, Song H, Xu Z, Cui J, Zhou M, Yang J, and Xiao J. 2017. A clinical and mechanistic study of topical borneol-induced analgesia. EMBO Mol Med 9:802-815. 10.15252/emmm.201607300

Xuefen CBXYYXDJY. 1991. Variations in chemical components of leaf oil from reproductive progenies of Cinnamomum tenuipilum. Acta Botanica Yunnanic 73:6.

Yang MY, Khine AA, Liu JW, Cheng HC, Hu A, Chen HP, and Shih TL. 2018. Resolution of isoborneol and its isomers by GC/MS to identify "synthetic" and "semi-synthetic" borneol products. Chirality 30:1233-1239. 10.1002/chir.23017

Yang T, Li J, Wang HX, and Zeng Y. 2005. A geraniol-synthase gene from Cinnamomum tenuipilum. Phytochemistry 66:285-293. 10.1016/j.phytochem.2004.12.004

Yin JL, Wong WS, Jang IC, and Chua NH. 2017. Co-expression of peppermint geranyl diphosphate synthase small subunit enhances monoterpene production in transgenic tobacco plants. New Phytol 213:1133-1144. $10.1111 / \mathrm{nph} .14280$

Zhou T, Luo X, Yu C, Zhang C, Zhang L, Song YB, Dong M, and Shen C. 2019. Transcriptome analyses provide insights into the expression pattern and sequence similarity of several taxol biosynthesis-related genes in three Taxus species. BMC Plant Biol 19:33. 10.1186/s12870-019-1645-x

Zou L, Zhang Y, Li W, Zhang J, Wang D, Fu J, and Wang P. 2017. Comparison of Chemical Profiles, AntiInflammatory Activity, and UPLC-Q-TOF/MS-Based Metabolomics in Endotoxic Fever Rats between Synthetic Borneol and Natural Borneol. Molecules 22. 10.3390/molecules22091446

Peer] reviewing PDF | (2019:11:43541:2:2:NEW 7 May 2020) 


\section{Table $\mathbf{1}$ (on next page)}

transcriptome sequencing statistics of $C$. burmanni with different borneol content 
Table 1 transcriptome sequencing statistics of $C$. burmanni with different borneol content

\begin{tabular}{llllllll}
\hline Sample & Raw reads & Clean reads & Clean bases & Q20(\%) & Q30(\%) & GC content (\%) & Mapped ratio \\
\hline HBT & $28,097,241$ & $28,097,241$ & $8.40 \mathrm{G}$ & 98.02 & 94.61 & 47.67 & $81.34 \%$ \\
LBT & $21,355,948$ & $21,355,948$ & $6.39 \mathrm{G}$ & 98.04 & 94.37 & 47.36 & $80.36 \%$ \\
BFT & $22,169,937$ & $22,169,937$ & $6.42 \mathrm{G}$ & 98.08 & 94.72 & 47.64 & $80.72 \%$ \\
Summary & $71,623,126$ & $71,623,126$ & $21.21 \mathrm{G}$ & - & - & 47.55 & - \\
\hline
\end{tabular}

2 a Q20 indicates the percentage of bases with a Phred value $>20$, while ${ }^{b} \mathrm{Q} 30$ indicates the percentage of bases with a Phred value $3>30$

4 


\section{Table 2 (on next page)}

Length distribution of transcripts and unigenes of the assembly transcritome 
Table 2 Length distribution of transcripts and unigenes of the assembly transcritome

\begin{tabular}{lll}
\hline Item & Transceript & Uinigene \\
\hline Total Number & 142,673 & 100,218 \\
Total Length(bp) & $133,416,440$ & $71,533,702$ \\
N50 Length(bp) & 1,625 & 1,182 \\
Mean Length(bp) & 935 & 713 \\
\hline
\end{tabular}

2 
Figure 1

Biosynthetic pathway of terpenes and biosynthetic steps from BPP to D-borneol.

a Biosynthetic pathway of terpenes; b Biosynthetic steps from BPP to D-borneol. Each solid arrow represents a biosynthetic reaction step.

a

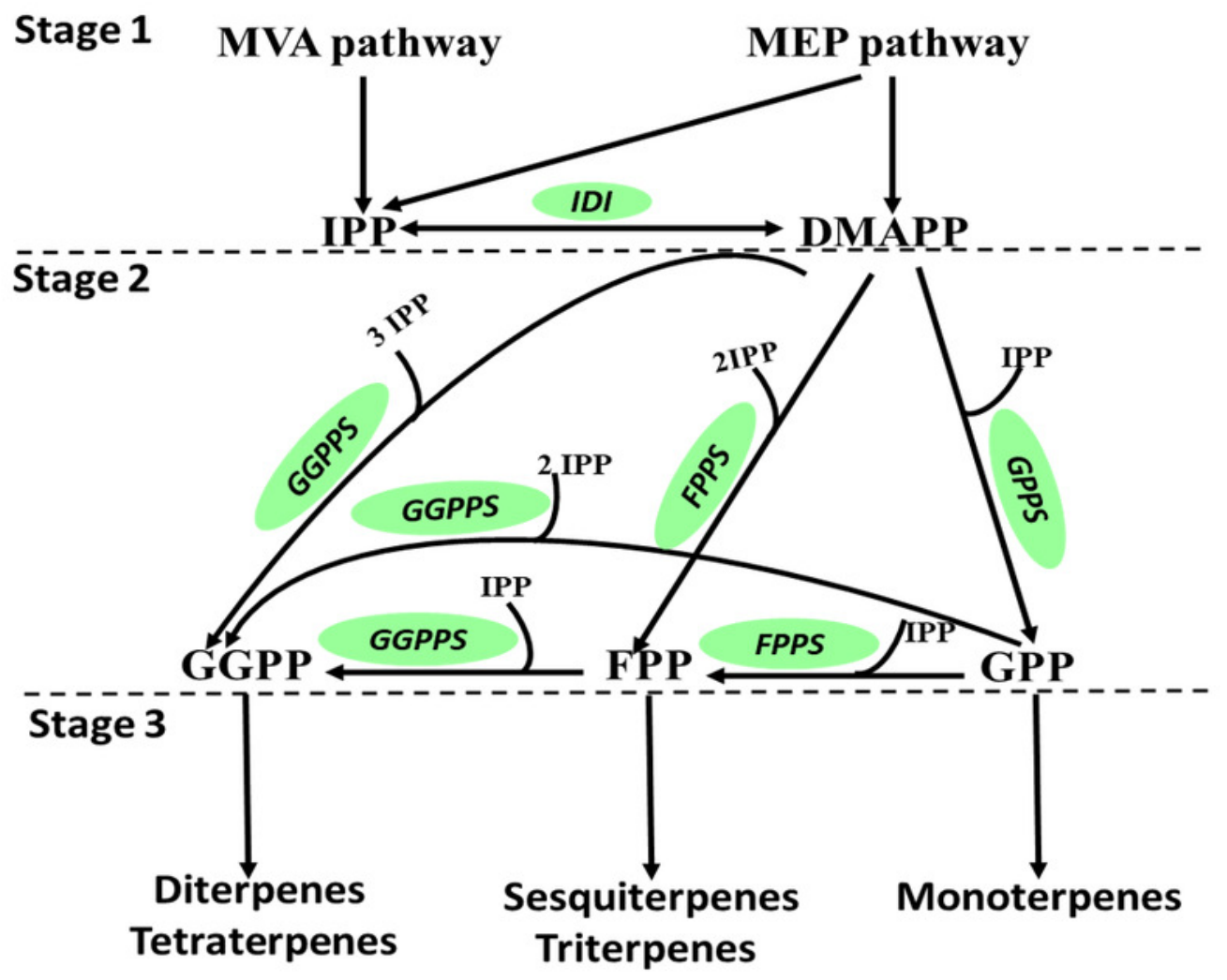

b

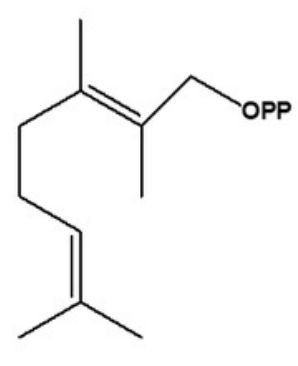

GPP
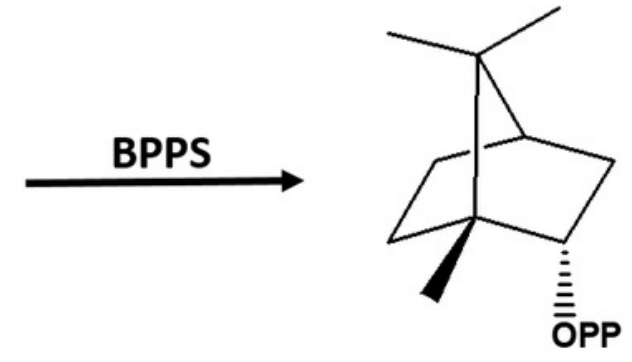

BPP
Phosphatase

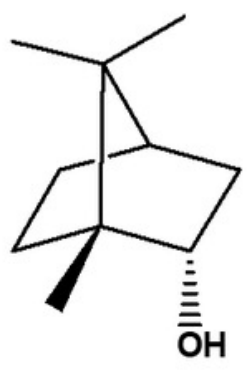

D-borneol 


\section{Figure 2}

Comparison of the content of D-borneol in different chemotype of $C$. burmannii.

a-c Leaf morphology of three different chemotypes of $C$. burmanni $\square$ while a for HBT, $b$ for LBT, c for BFT; d-h GC/MS peak of the C. burmannii with different D-borneol content. d BFT ; e LBT; f HBT; g D-borneol ; h blank (Petroleum ether) ; i Mass spectra of D-borneol from C. burmannii sample; $\mathrm{j}$ Mass spectra of D-borneol standard. $\mathrm{k}$ the content of $\mathrm{D}$-borneol were quantified by GC/MS method. ND: not detected. I Percentage of terpenes in HBT. $m$ Percentage of terpenes in LBT. $n$ Percentage of terpenes in BFT. 

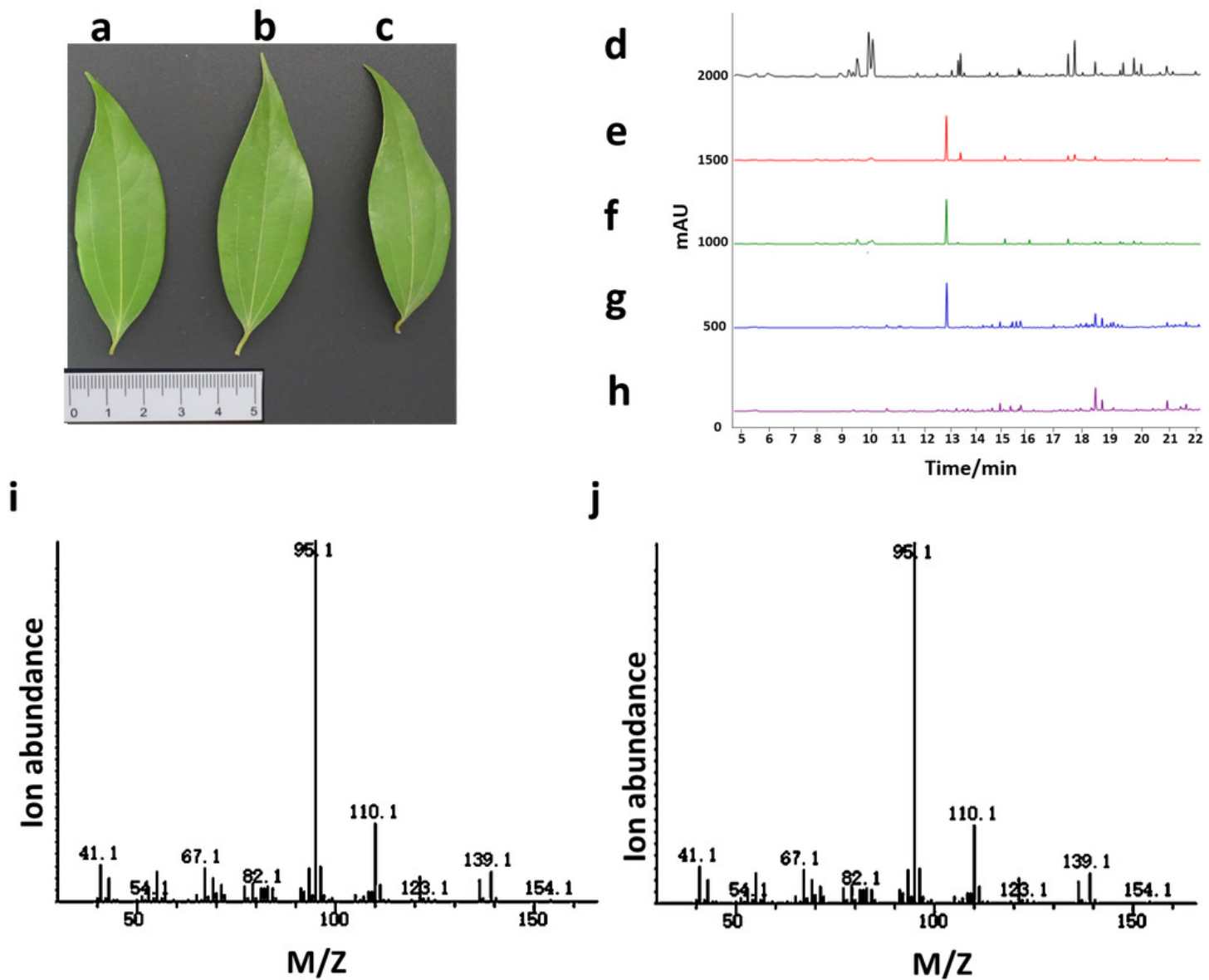

k

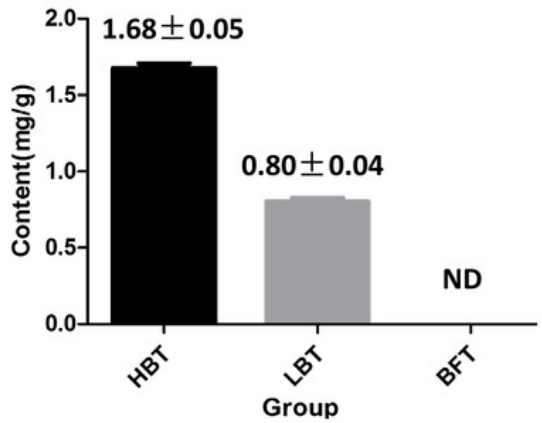

I
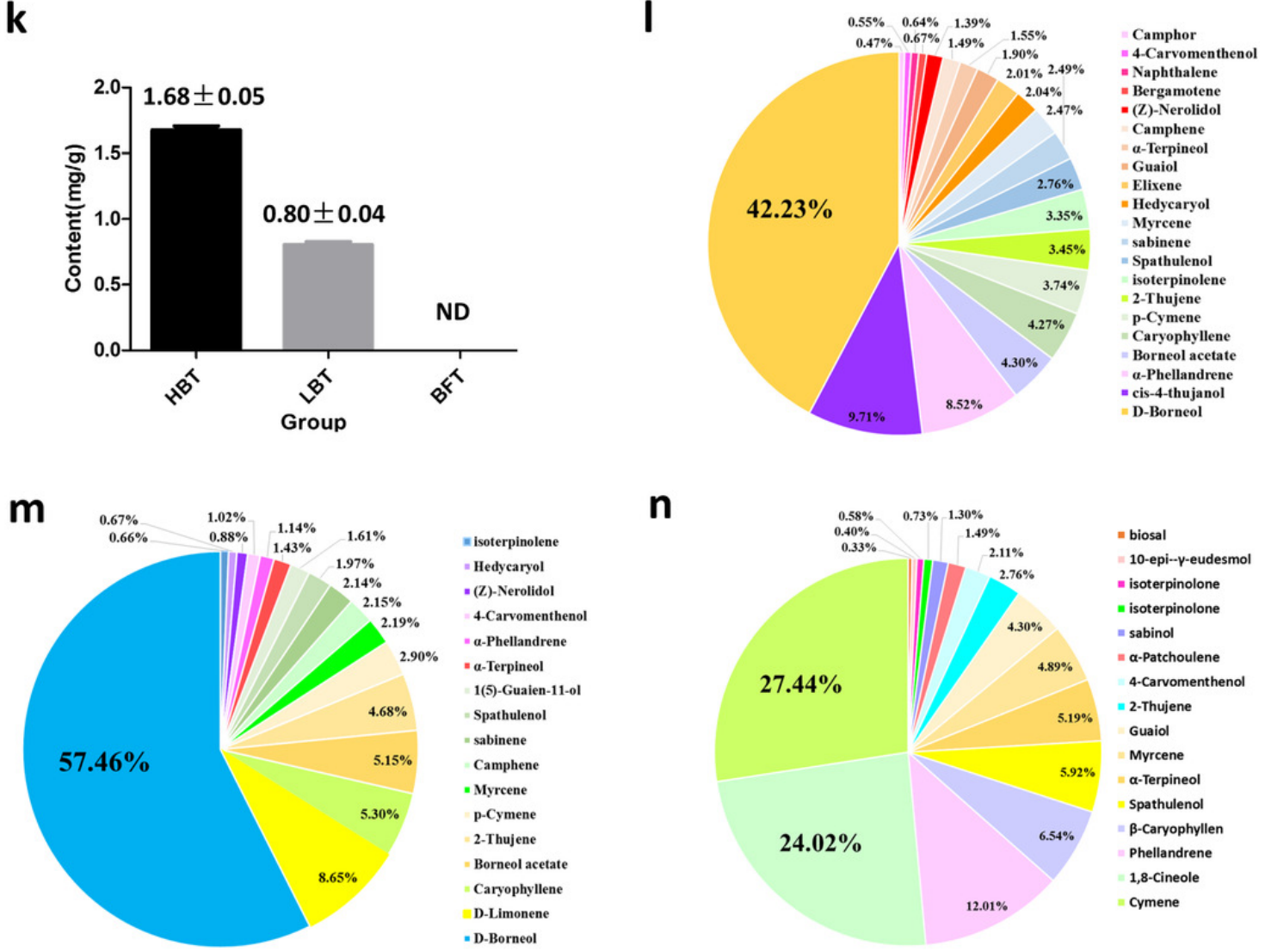

n

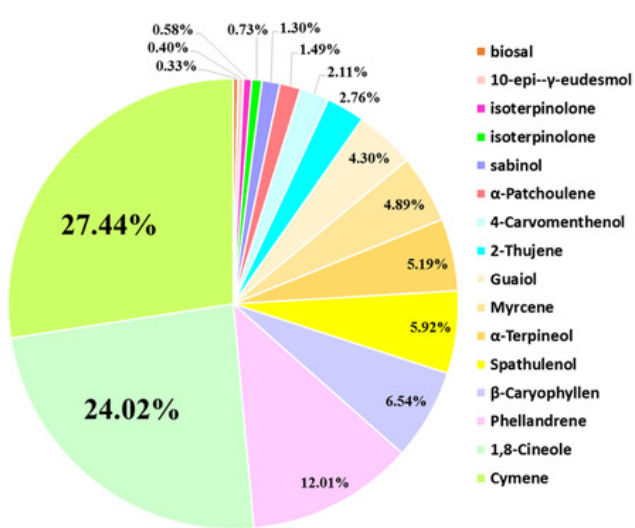




\section{Figure 3}

Transcriptome assembly, annotation results and identification of the DEGs among $C$. burmanni with different borneol content. a The length distribution of unigenes and transcripts of $C$. burmanni. b The annotation of unigenes based different data

a The length distribution of unigenes and transcripts of $C$. burmanni. b The annotation of unigenes based different databases. c Venn diagram of the distribution of annotation information from different public databases. $d$ The species distribution of the annotated unigenes. e Hierarchical clustering of DEGs among $\mathrm{C}$. burmanni with different borneol content. $\mathrm{f}$ The DEGs were clustered into four clusters by K-means clustering, based on the Pearson correlation distances. g Venn-diagram of significantly different DEGs. h Number of up- and down-regulated DEGs. 
a

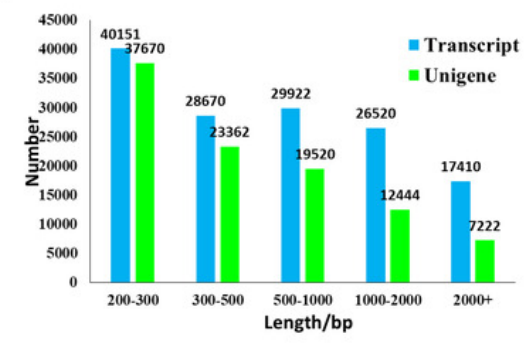

C

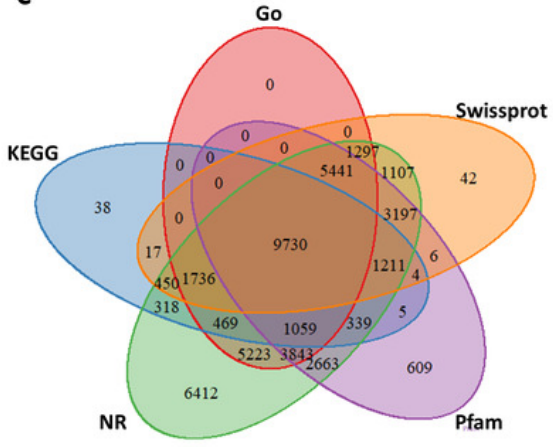

e

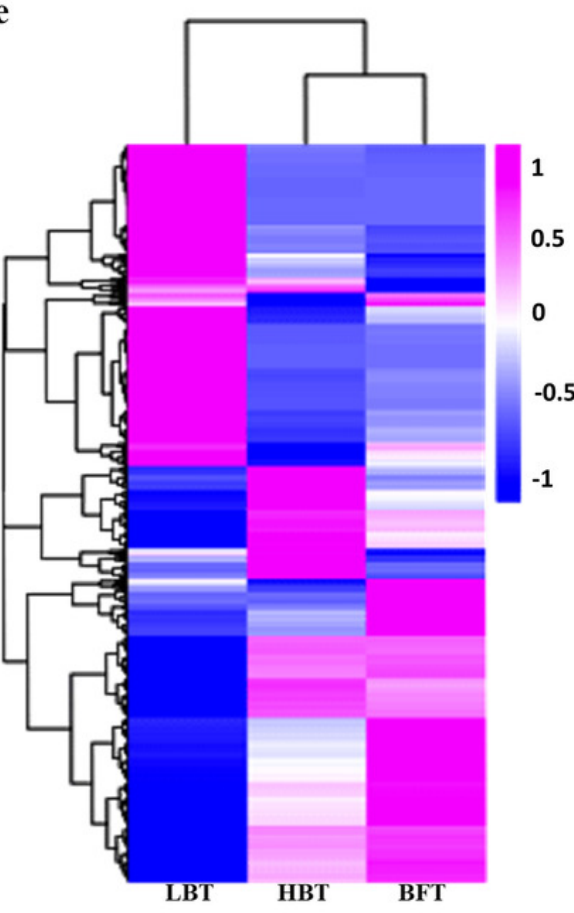

g

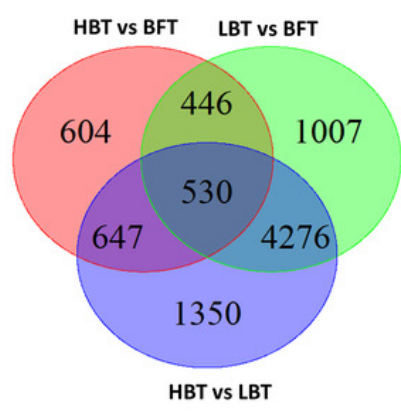

b

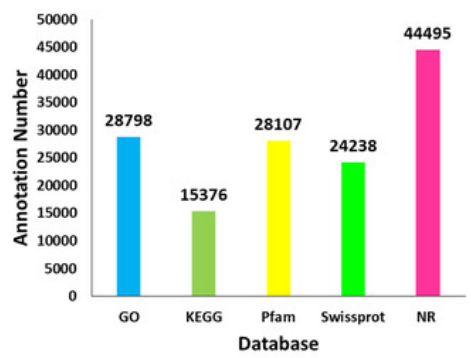

d

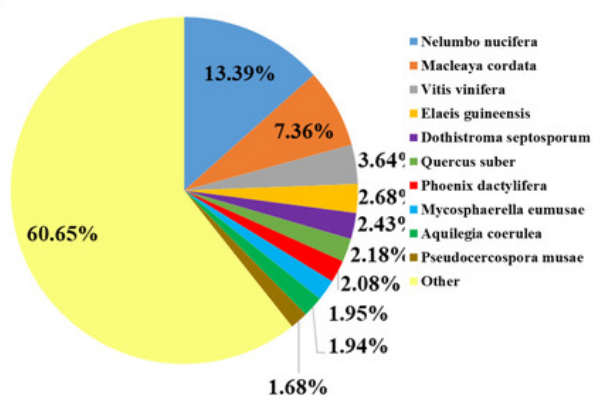

f
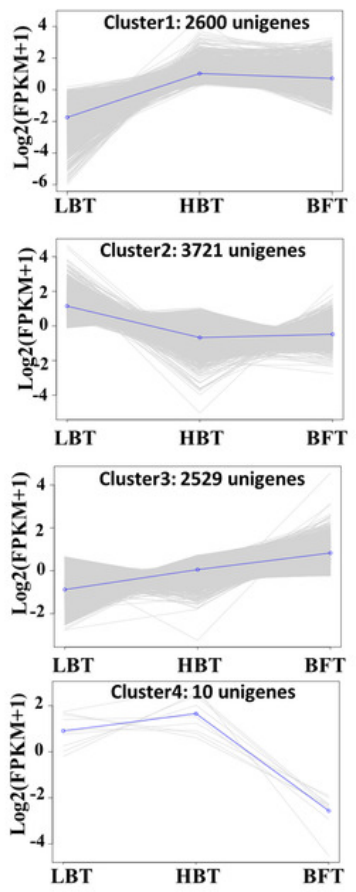

h

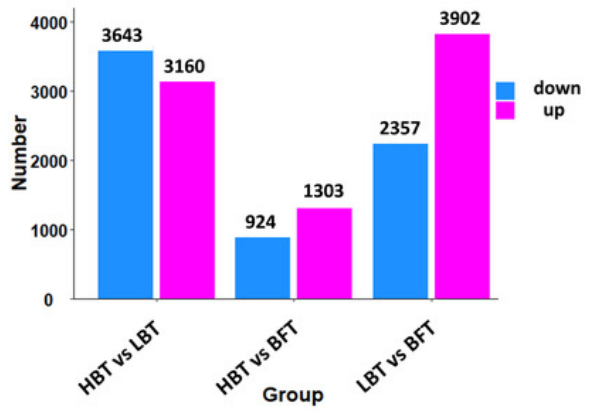


Figure 4

Functional enrichment analysis of the DEGs among $C$. burmanni with different borneol content.

a KEGG enrichment analysis of DEGs in the three comparisons. b GO enrichment analysis of DEGs in the three comparisons. 
a

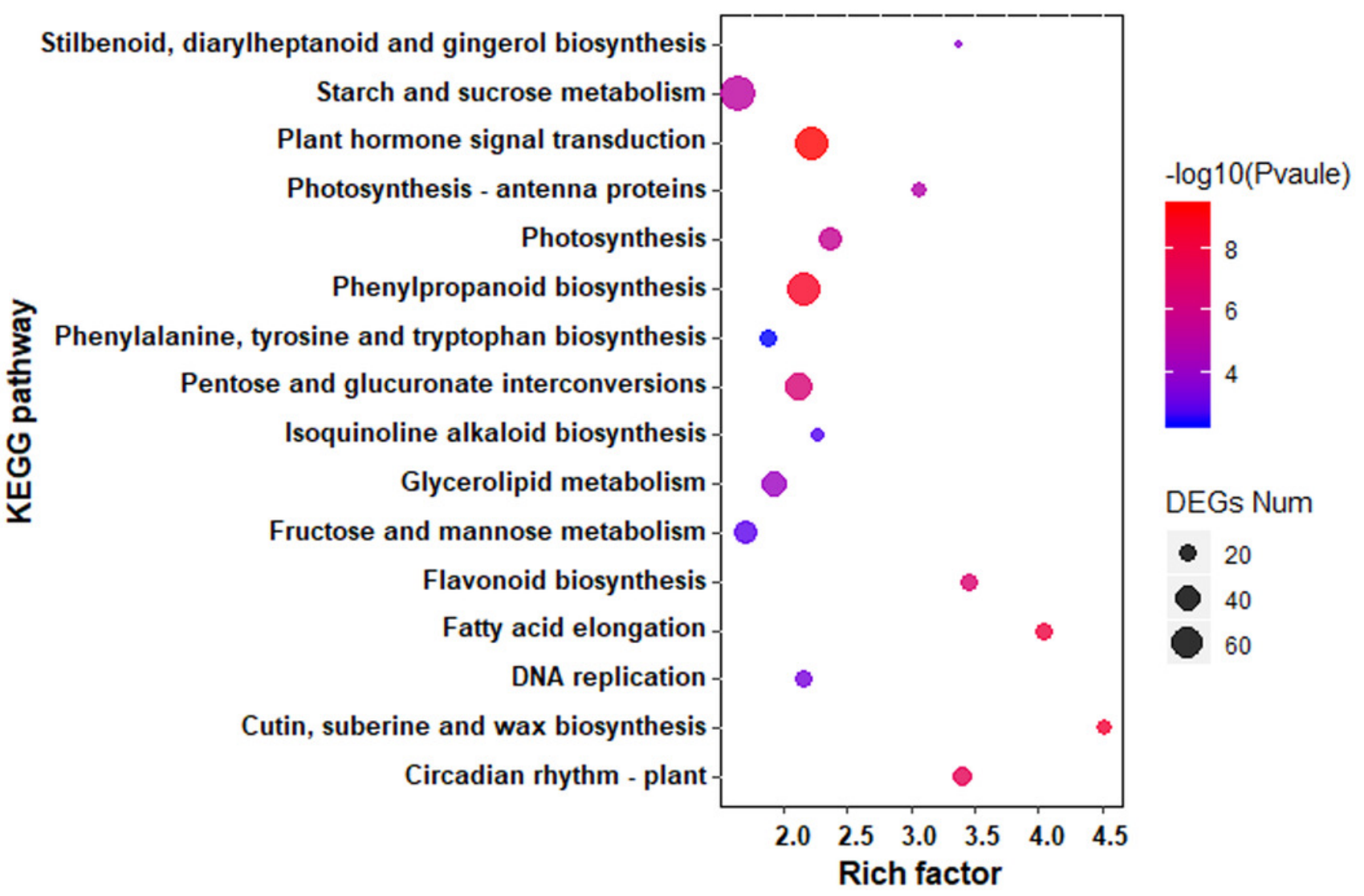

b

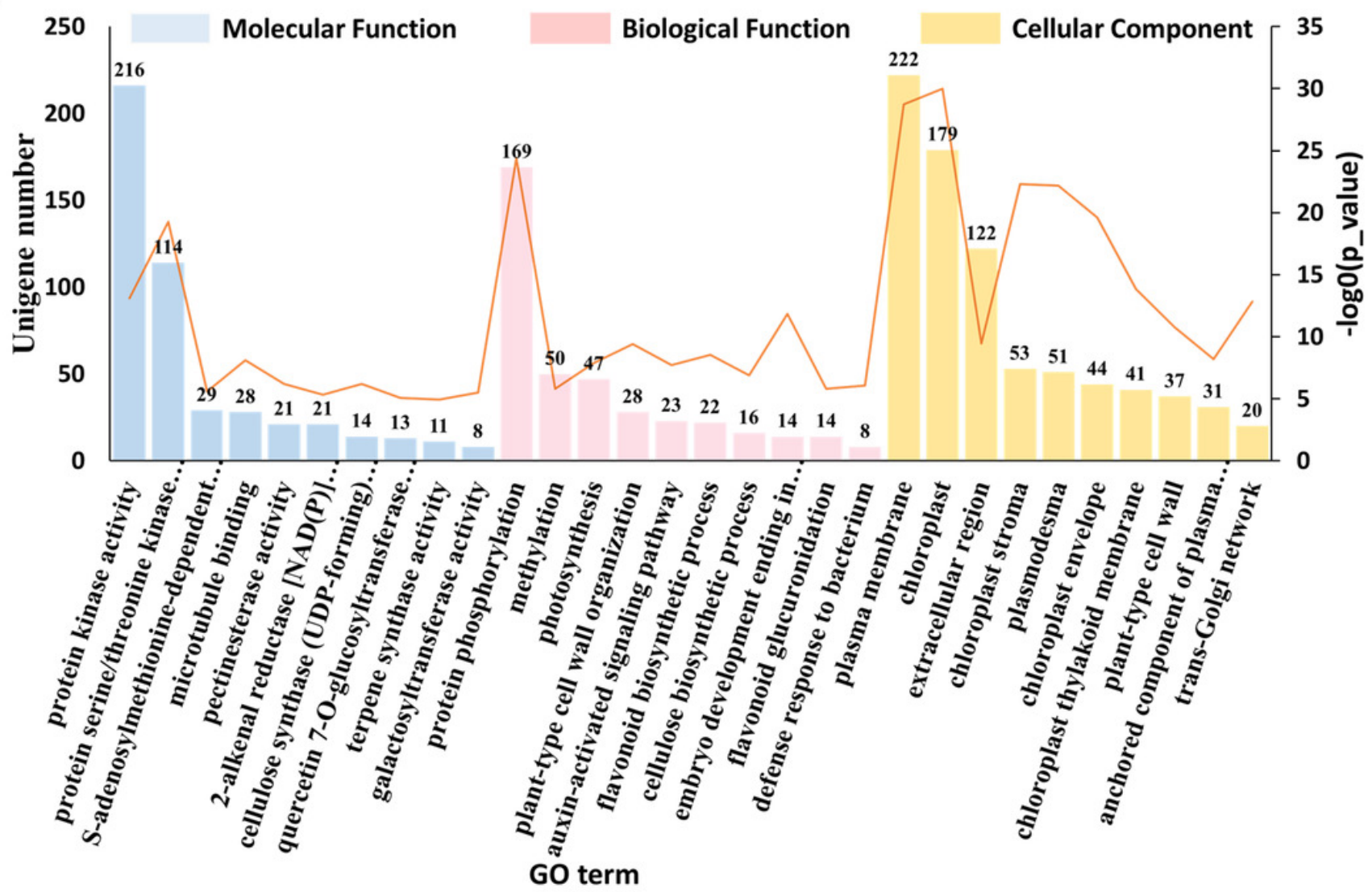




\section{Figure 5}

Genes related to the monoterpenoid biosynthesis pathways in C. burmanni and Candidate transcriptional factors analysis

.a brief schematic of monoterpenoid biosynthesis pathway which was modified from KEGG databases. The red box represents the enzyme. DXS: 1-Deoxy-D-xylulose-5-phosphate synthase. DXR: 1-Deoxy-D-xylulose 5-phosphate reductoisome-rase. MCT: 2-C-methyl-Derythritol 4-phosphate cytidylyltransferase. CMK: 4-(Cytidine-5-dipho-spho)-2-C-methyl-D-erythritol kinase. MDS: 2-C-methyl -D-erythritol 2,4-cyclodiphosphate synthase. HDS: 4Hydroxy-3-methylbut-2 -enyl-diphosphate synthase. HDR: 4-Hydroxy-3-meth-ylbut-2-enyl diphosphate reductase. IPPI: isopentenyl diphosphate isomerase. GPPS: geranyl diphosphate synthase. DXP: 1-Deoxy-D-xylulose 5-phosphate. MEP: 2-C-Methyl-D-erythritol 4-phosphate. CDP-ME: 4-(Cytidine 5'-diphospho)-2-C-methyl-D-erythritol. ME-CPP: 2-Phospho-4-(cytidine 5'diphospho)-2-C-methyl-D-erythritol. CDI: 2-C-Methyl-D-erythritol2,4-cyclodiphosphate. HMBPP: 1-Hydroxy-2-methyl-2-butenyl 4-diphosphate. $\mathbf{b}$ The expression level of the unigenes and the results were shown as a heatmap. c The top $20 \mathrm{TF}$ families with the largest number of genes. $\mathbf{d}$ expression level of the candidate BZIP transcription factors. e expression level of the WRKY transcriptional factors. $\mathbf{f}$ expression level of the BHLH transcriptional factors. $\mathbf{g}$ expression level of the ERF transcriptional factors. $\mathbf{h}$ expression level of the MYB transcriptional factorsa 
a

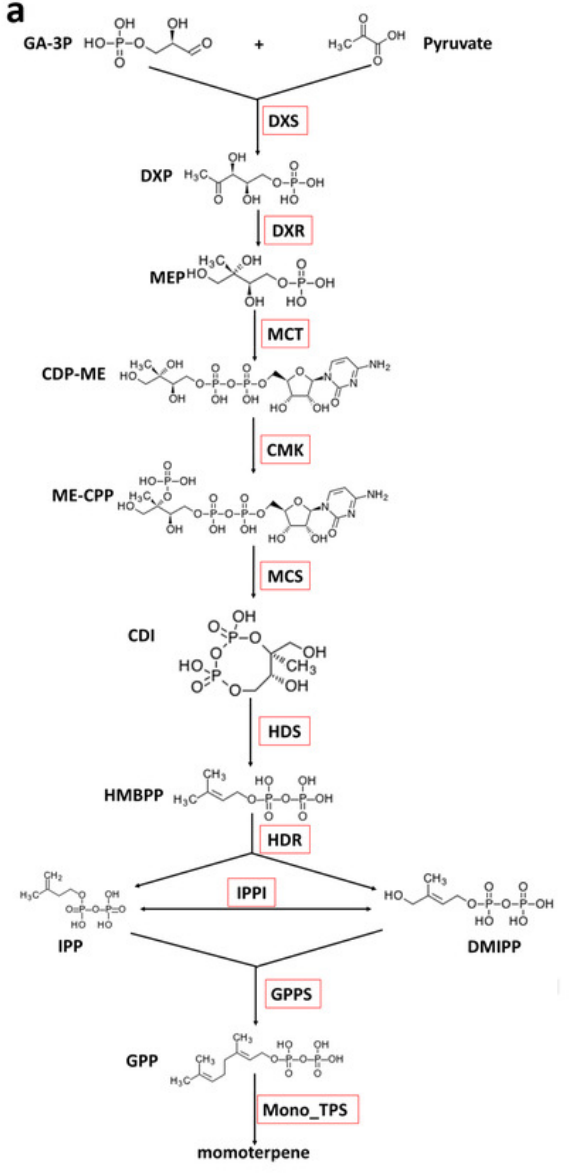

c

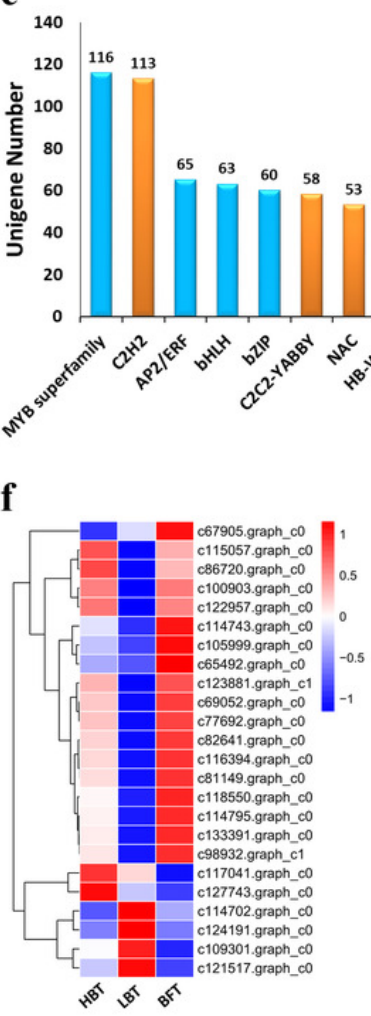

b

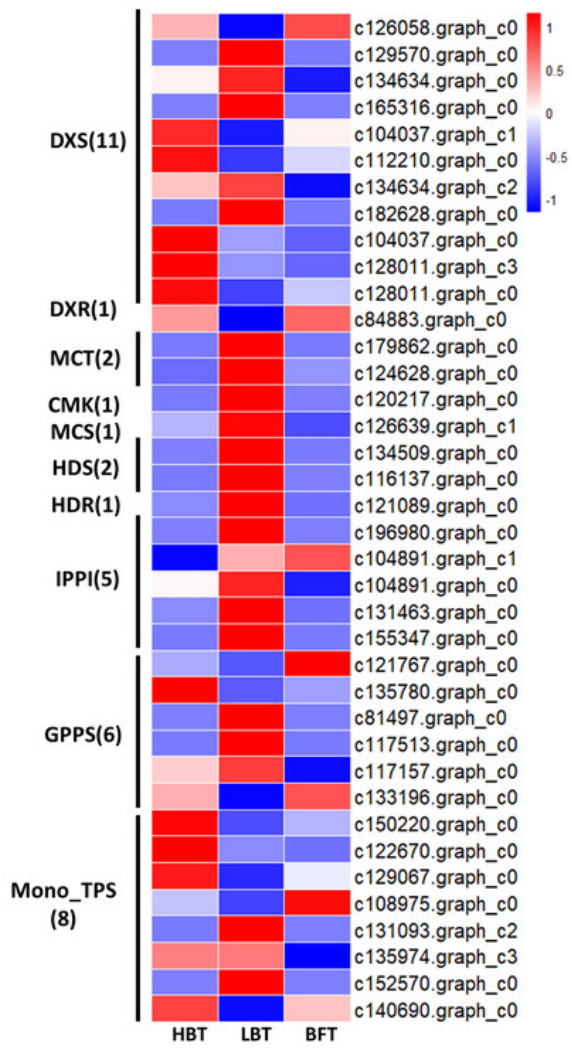

d

TF family name

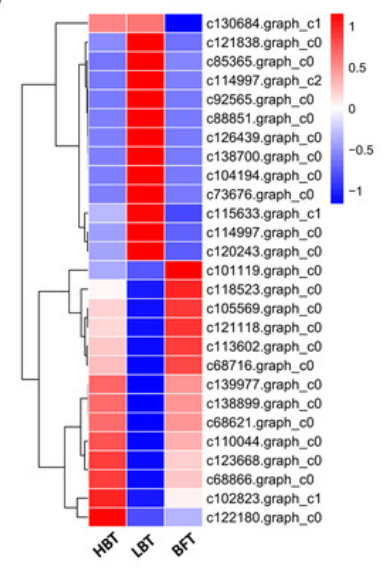

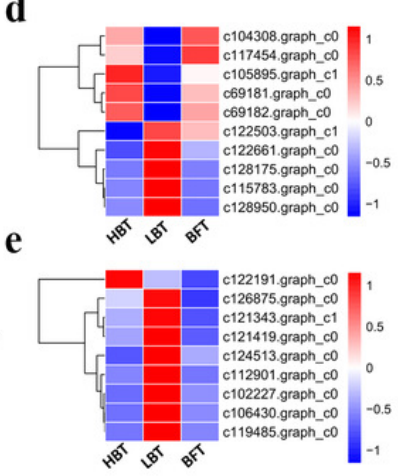

h

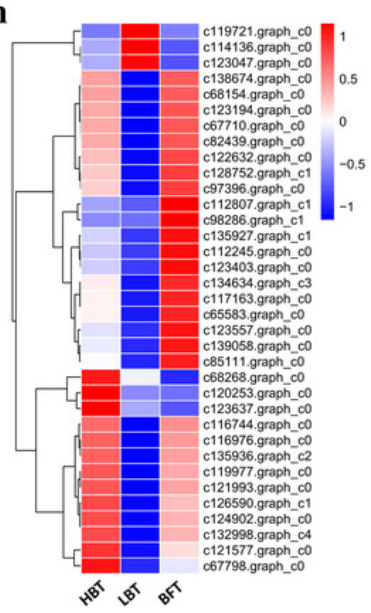




\section{Figure 6}

qRT-PCR validation of genes related to the biosynthesis of terpene from the RNA-seq data.

a-0: The FPKM values and the qRT-PCR for the 15 genes selected for this study. The FPKM data of the unigenes were shown in pink bars, while the blue bars indicated the GPCR results. The left $\mathrm{Y}$-axis represents the relative expression calculated by $\mathrm{qRCP}$, and the right $\mathrm{Y}$-axis represents the FPKM value of the RNA sequencing data. 
a

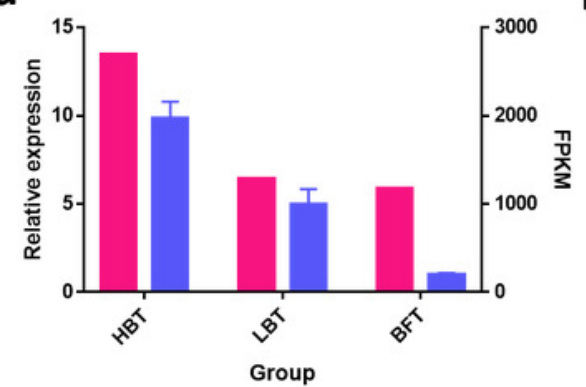

d

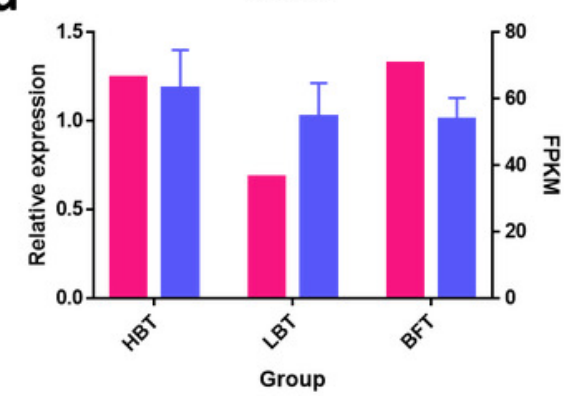

g

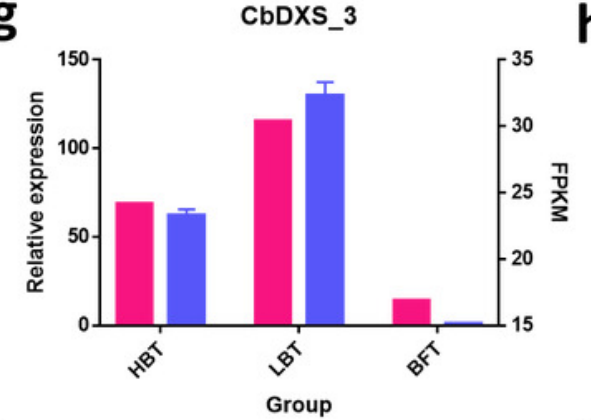

j

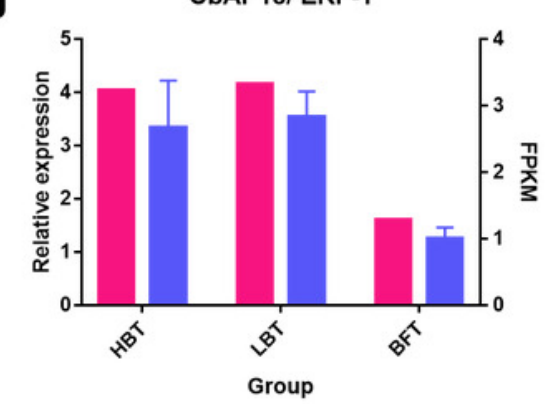

m

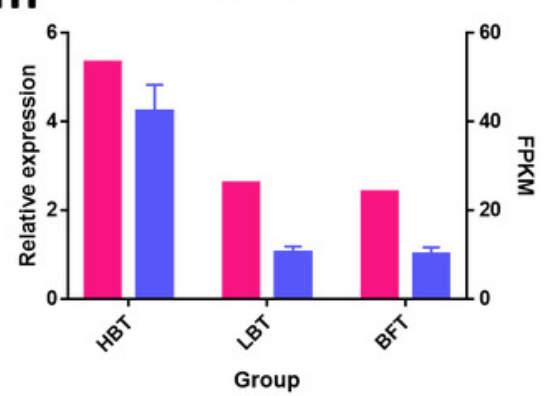

b

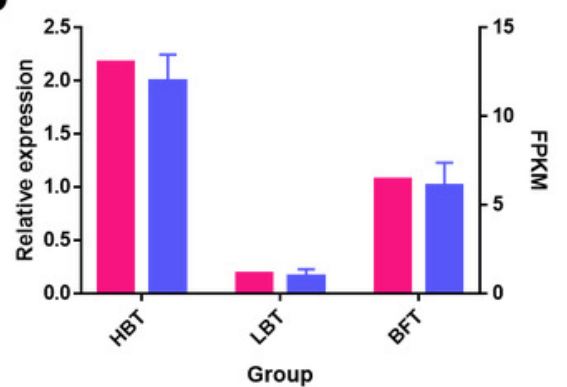

e

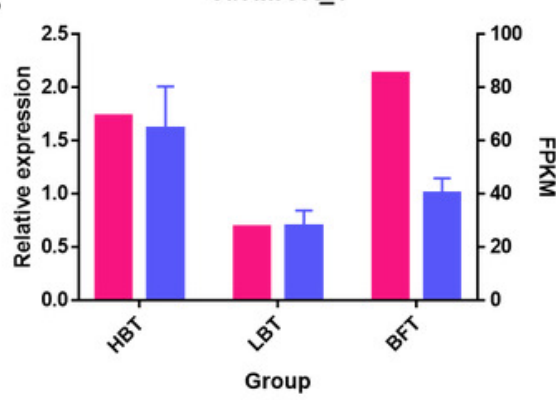

h

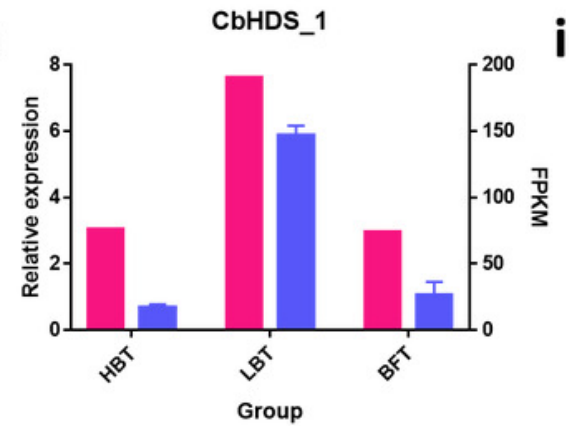

k

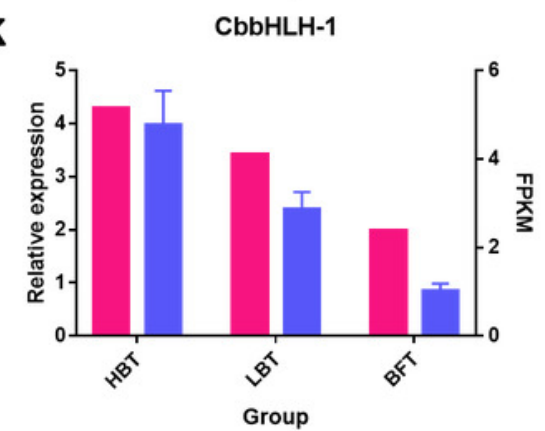

n

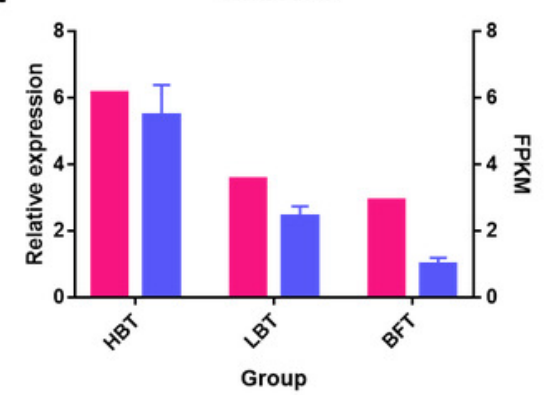

C

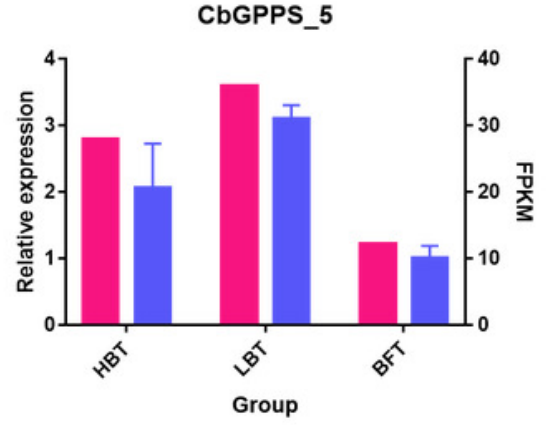

f

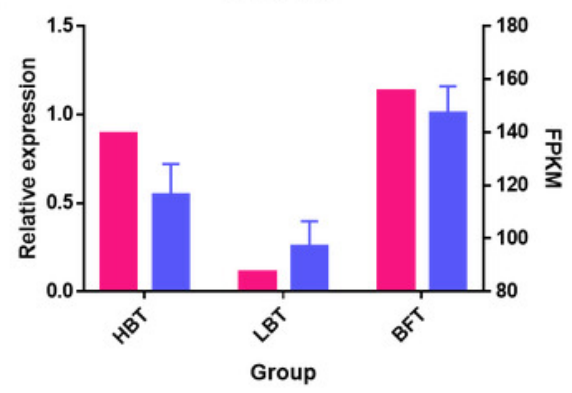

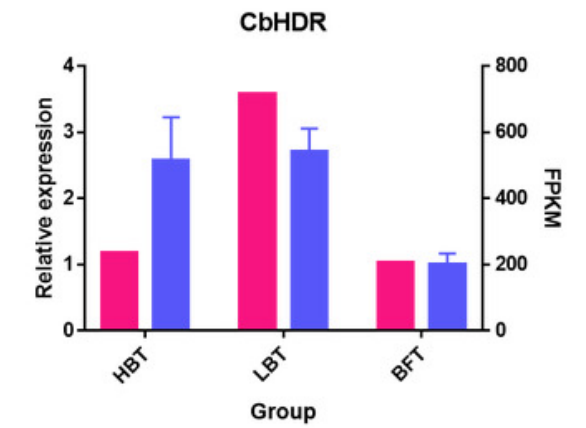

I CbbHLH-2

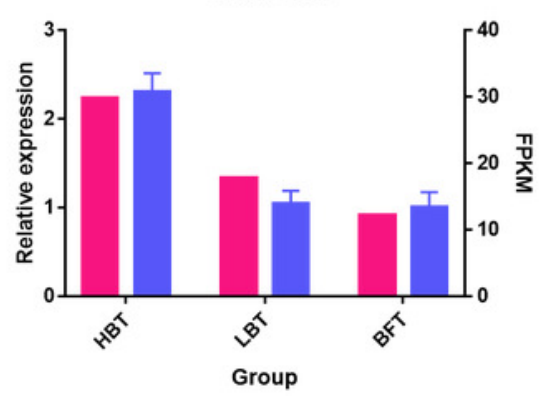

o

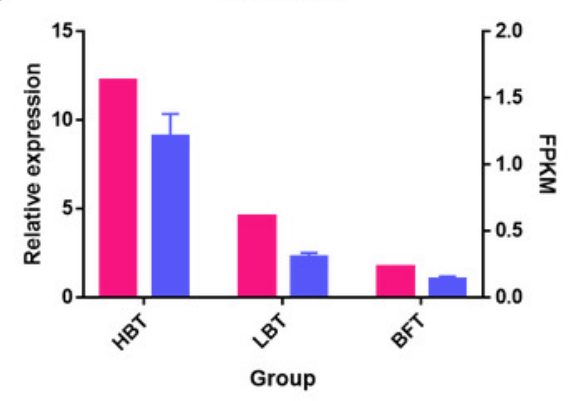

